\title{
Associative algebras for (logarithmic) twisted modules for a vertex operator algebra
}

\author{
Yi-Zhi Huang and Jinwei Yang
}

\begin{abstract}
We construct two associative algebras from a vertex operator algebra $V$ and a general automorphism $g$ of $V$. The first, called $g$-twisted zero-mode algebra, is a subquotient of what we call $g$-twisted universal enveloping algebra of $V$. These algebras are generalizations of the corresponding algebras introduced and studied by FrenkelZhu and Nagatomo-Tsuchiya in the (untwisted) case that $g$ is the identity. The other is a generalization of the $g$-twisted version of Zhu's algebra for suitable $g$-twisted modules constructed by Dong-Li-Mason when the order of $g$ is finite. We are mainly interested in $g$-twisted $V$-modules introduced by the first author in the case that $g$ is of infinite order and does not act on $V$ semisimply. In this case, twisted vertex operators in general involve the logarithm of the variable. We construct functors between categories of suitable modules for these associative algebras and categories of suitable (logarithmic) $g$-twisted $V$-modules. Using these functors, we prove that the $g$-twisted zero-mode algebra and the $g$-twisted generalization of Zhu's algebra are in fact isomorphic.
\end{abstract}

\section{Introduction}

Orbifold conformal field theories play an important role in mathematics and physics. They are examples of conformal field theories constructed from known conformal field theories and automorphisms of these known ones. Mathematically, the study of orbifold conformal field theories can be reduced to the study of the twisted representation theory of vertex operator algebras, that is, the theory of representations of vertex operator algebras twisted by automorphisms. In fact, the first example of orbifold conformal field theories is the conformal field theory corresponding to the moonshine module vertex operator algebra constructed by Frenkel, Lepowsky and Meurman [FLM]. One conjecture proposed by the first author in this twisted representation theory of vertex operator algebras is that $g$-twisted modules for a vertex operator algebra $V$ satisfying suitable conditions and elements $g$ of a group $G$ of automorphisms of $V$ equipped with twisted (logarithmic) intertwining operators among $g$ twisted modules for $g \in G$ have a structure of $G$-equivariant intertwining operator algebras satisfying a modular invariance property. This conjecture in fact implies that the category of $g$-twisted $V$-modules for $g \in G$ has a structure of $G$-crossed (tensor) category in the sense of Turaev [T]. 
Automorphisms of a vertex operator algebra can be of finite or infinite orders. An element of the Monster group gives an automorphism of the moonshine module vertex operator algebra [FLM]. Such an automorphism is of finite order. An element of a simply connected finite-dimensional Lie group gives an automorphism of the vertex operator algebra associated to the affine Lie algebra of the Lie algebra of the Lie group [FLM] [FZ]. Such an automorphism is in general of infinite order. Moreover, in general it might not act on the vertex operator algebra semisimply.

Twisted modules for a vertex operator algebra associated to an automorphism of finite orders were introduced in the construction of the moonshine module vertex operator algebra in [FLM]. In [H3], the first author introduced a notion of twisted module associated to a general automorphism whose order does not have to be finite. One important new feature of twisted modules in the general case is that twisted vertex operators might involve the logarithm of the variable (logarithmic $g$-twisted module). For a historical discussion of twisted modules for vertex operator algebras and the relevant references, see [H3]. See also [B] for the Jacobi identity for a suitable component of the twisted vertex operators that we shall derive from the axioms in [H3] and use in this paper.

In the representation theory of Lie algebras, one of the most important tools is the universal enveloping algebra of a Lie algebra. The representation theory of a Lie algebra is equivalent to the representation theory of its universal enveloping algebra. It is natural to try to find associative algebras that play the similar roles in the representation theory of vertex operator algebras.

Let $V$ be a vertex operator algebra. Frenkel and Zhu in [FZ] first constructed the universal enveloping algebra $U(V)$ of $V$. $V$-modules of the weakest type (even more general than the so called weak $V$ modules) are equivalent to $U(V)$-modules. Though $U(V)$ is very natural, it is not very useful because in the representation theory of vertex operator algebras, we are interested mostly in $V$-modules with suitable lower bounded gradings. In [Z], Zhu constructed an associative algebra $A(V)$ on a quotient of $V$ with the product obtained from the vertex operator map. He also constructed implicitly functors between the category of $V$-modules with suitable lower bounded gradings and the category of $A(V)$-modules. In [H2], motivated by the geometry of vertex operator algebras, the first author constructed an associative algebra $\tilde{A}(V)$ using a product and a quotient that look very different from those defining $A(V)$. It was also proved in [H2] that $\tilde{A}(V)$ is in fact isomorphic to $A(V)$ by constructing explicitly an isomorphism between them using the conformal transformation $\frac{1}{2 \pi i} \log (1+2 \pi i z)$.

To study twisted modules for vertex operator algebras, we would also like to find suitable associative algebras such that the study of twisted modules can be reduced to the study of modules for these associative algebras. In the case that the automorphism $g$ of the vertex operator algebra $V$ is of finite order, Dong, Li and Mason in [DLM1] generalized Zhu's construction to obtain an associative algebra $A_{g}(V)$ together with functors between the categories of suitable $g$-twisted $V$-modules and $A_{g}(V)$-modules. This associative algebra $A_{g}(V)$ is in fact a subalgebra of Zhu's algebra for the fixed point vertex operator algebra under $g$. The construction in [DLM1] cannot be generalized directly to the case that the 
order of $g$ is infinite.

In the present paper, for a vertex operator algebra $V$ and an automorphism $g$ of $V$, we first construct a $g$-twisted universal enveloping algebra $U_{g}(V)$ such that $g$-twisted $V$-modules of the weakest type (even more general than $g$-twisted weak $V$-modules) are equivalent to $U_{g}(V)$-modules. The construction is a straightforward generalization of the construction of Frenkel-Zhu to the twisted case. We take the tensor algebra of the affinization of $V$ with suitable powers of the variable and take a topological completion so that suitable infinite sums are allowed, and then divide this algebra by all the relations corresponding to the identities that should hold for any type of $g$-twisted modules.

Just as in the case of $V$-modules, $U_{g}(V)$ is not very useful because we are interested mostly in $g$-twisted $V$-modules with suitable lower bounded gradings. For $g$-twisted $V$ modules graded by conformal weights, there exist lowest weight spaces. In the case that such a $g$-twisted $V$-module is irreducible, the lowest weight space determines the module completely. It is natural to expect that there exists an associative algebra obtained from $U_{g}(V)$ such that the lowest weight space of an irreducible $g$-twisted $V$-module is a module for this algebra.

In this paper, using $U_{g}(V)$, we construct an associative algebra $Z_{g}(V)$ satisfying this property and call it $g$-twisted zero-mode algebra, although the more appropriate name for this algebra is probably $g$-twisted imaginary-mode algebra or $g$-twisted zero-real-mode algebra. In fact, $U_{g}(V)$ is graded by conformal weights. Take the subalgebra of $U_{g}(V)$ generated by elements of imaginary weights and then take the quotient of this algebra by the ideal generated by elements of the form $u v$ where $u, v \in U_{g}(V)$ have weights $k+m$ and $-m$ for $k \in \mathbb{I}$ (the set of imaginary numbers) and $m \in \mathbb{C}$ satisfying $\Re(m)>0$. This quotient algebra is our $g$-twisted zero-mode algebra $Z_{g}(V)$.

We also construct an associative algebra $\tilde{A}_{g}(V)$, generalizing the algebra $\tilde{A}(V)$ in [H2], and an isomorphic algebra $A_{g}(V)$, generalizing the algebra constructed in [DLM1] for $g$ of finite order. In fact, in this case, $\tilde{A}_{g}(V)$ is more natural to work with. We give our constructions and proofs mostly for $\tilde{A}_{g}(V)$. The construction and results for $A_{g}(V)$ can be easily derived from those for $\tilde{A}_{g}(V)$ using the isomorphism between them. One interesting feature in the case that the order of $g$ is infinite is that $A_{g}(V)$ is in general not a subalgebra of Zhu's algebra for the fixed point vertex operator subalgebra under $g$. It is instead a subalgebra of Zhu's algebra for the fixed point vertex operator subalgebra under the semisimple part of $g$.

Our main results in this paper are the constructions of functors between the categories of suitable $g$-twisted $V$-modules, suitable $Z_{g}(V)$-modules and suitable $\tilde{A}_{g}(V)$-modules (or $A_{g}(V)$-modules). As in the case that the order of $g$ is finite, a suitable $g$-twisted $V$-module $W$ has a subspace $\Omega_{g}(W)$ on which the components of the twisted vertex operators whose weights have negative real parts act as 0 . It is easy to prove from the definitions that $\Omega_{g}(W)$ is a $Z_{g}(V)-, \tilde{A}_{g}(V)$ - or $A_{g}(V)$-module. We then construct right inverses of these functors and derive some consequences that will be useful for future study. Finally, using these results, we prove that $Z_{g}(V), \tilde{A}_{g}(V)$ and $A_{g}(V)$ are isomorphic to each other.

In the case that $g$ is the identity, the $g$-twisted zero-mode algebra becomes the zeromode algebra, which appeared first in physics (see [BN]) and introduced rigorously in [FZ]. 
In [NT], Nagatomo and Tsuchiya [NT] proved that the zero-mode algebra is isomorphic to Zhu's algebra. As a special case (the case that $g$ is the identity), our proof that $Z_{g}(V)$ and $A_{g}(V)$ are isomorphic gives in particular a different proof that the zero-mode algebra is isomorphic to Zhu's algebra.

It seems to be easier to calculate $Z_{g}(V)$ than to calculate $A_{g}(V)$ or $\tilde{A}_{g}(V)$. On the other hand, $\tilde{A}_{g}(V)$ is more natural for the study of modular invariance. $A_{g}(V)$ is in some sense a bridge connecting them. We expect that $Z_{g}(V)$ will be especially useful for the construction and study of twisted modules and twisted intertwining operators.

There are also higher zero-mode algebras $Z_{g, n}(V)$ for $n \in \mathbb{N}$ as the quotient of the subalgebra of $U_{g}(V)$ generated by elements of imaginary weights by the ideal generated by elements of the form $u v$ where $u, v \in U_{g}(V)$ have weights $k+m$ and $-m$ for $k \in \mathbb{I}$ and $m \in \mathbb{C}$ satisfying $\Re(m)>n$. The $g$-twisted zero-mode algebra $Z_{g}$ discussed above is in fact $Z_{g, 0}(V)$. We can also generalize higher Zhu's algebras $A_{n}(V)$ introduced by Dong, Li and Mason in [DLM2] to associative algebras $A_{g, n}(V)$ for $n \in \mathbb{N}$ such that $A_{g, 0}(V)=A_{g}(V)$ and $A_{1, n}(V)=A_{n}(V)$ when $g$ is the identity 1 . There are also $\tilde{A}_{g, n}(V)$ for $n \in \mathbb{N}$ generalizing $\tilde{A}_{g}(V)$. These algebras will be important for the study of twisted logarithmic intertwining operators. But for simplicity, we shall study only $Z_{g}(V), \tilde{A}_{g}(V)$ and $A_{g}(V)$ in this paper. The general case will be discussed in another paper.

The definition of $g$-twisted module in [H3] was formulated using the duality properties of the twisted vertex operators. It should be possible to use the duality properties and immediate consequences to construct the associative algebras and functors above (see [HY] for a construction of the functors between the category of modules for Zhu's algebra $A(V)$ and the category of suitable $V$-modules without using the commutator formula for modules). On the other hand, since twisted vertex operators are special (logarithmic) intertwining operators, suitable components of twisted vertex operators might satisfy some Jacobi-type identity as is discussed for intertwining operators by the first author in [H1]. A Jacobi-type identity will give formulas that can be used to simplify our constructions. Indeed, a Jacobi identity for suitable components of twisted vertex operators was obtained by Bakalov in $[\mathrm{B}]$. Bakalov gave in [B] a different definition of $g$-twisted $V$-module based on an associator formula for the twisted vertex operators. From this definition, he derived a Jacobi identity for the map obtained by taking the non-logarithmic component of the twisted vertex operator map, that is, the constant term in the twisted vertex operator map when we view the twisted vertex operator map as a polynomial in the logarithm of the variable. It was stated in [B] that a $g$-twisted $V$-module satisfying the original definition given by the first author in [H3] indeed satisfies the definition given in $[B]$. But no proof was given there.

Note that the twisted vertex operators given in the definition in [H3] are the objects that we are interested in orbifold conformal field theory, not their non-logarithmic components. For example, in the future, we would like to prove the modular invariance for the space of $q$-(pseudo-)traces of twisted vertex operators for $g$-twisted $V$-modules, not for the space of $q$-(pseudo-)traces of non-logarithmic components of twisted vertex operators for $g$-twisted $V$-modules. Thus before we can use the Jacobi identity in [B], we need to first show that for suitable $g$-twisted $V$-modules, these two definitions are equivalent. We give a proof of this 
equivalence in this paper. We then use freely the formulas derived from this Jacobi identity to give our constructions and proofs.

The present paper is organized as follows: In Section 2, we recall several variants of the notion of $g$-twisted $V$-module. We derive Bakalov's Jacobi identity for the non-logarithmic components of twisted vertex operators and some other useful properties in this section. In this section, we also prove that when the other conditions for (generalized) $g$-twisted $V$ modules hold, the duality property for the twisted vertex operators given in [H3] is equivalent to the Jacobi identity for non-logarithmic components of twisted vertex operators given in [B]. We give the constructions of $g$-twisted universal enveloping algebra $U_{g}(V)$ and the zeromode algebra $Z_{g}(V)$ in Section 3. The constructions of $\tilde{A}_{g}(V)$ and $A_{g}(V)$ are given in Section 4. Our main results, the constructions of functors between different categories, are given in Section 5. In Section 6, we give the proof that $Z_{g}(V), A_{g}(V)$ and $\tilde{A}_{g}(V)$ are isomorphic to each other.

Acknowledgments We would like to thank Robert McRae for comments on the formulation of the duality property in the definition of twisted modules.

\section{Twisted modules}

In this paper, we fix a vertex operator algebra $(V, Y, \mathbf{1}, \omega)$ and an automorphism $g$ of $V$. We shall use the convention that $\log z=\log |z|+\arg z \sqrt{-1}$ where $0 \leq \arg z<2 \pi$. We shall also use $l_{p}(z)$ to denote $\log z+2 \pi p \sqrt{-1}$ for $p \in \mathbb{Z}$. Besides the usual notations $\mathbb{C}, \mathbb{R}, \mathbb{Z}, \mathbb{Z}_{+}$ and $\mathbb{N}$ for the sets of complex numbers, real numbers, integers, positive integers and natural numbers, respectively, we also use $\mathbb{I}$ and $\overline{\mathbb{C}}_{+}$to denote the set of imaginary numbers and the closed right half complex plane.

In this section, we recall the definition of several variants of the notion of $g$-twisted $V$ module and prove a number of useful properties for these $g$-twisted $V$-modules. Many of the formulas proved in this section were obtained first by Bakalov $[B]$ from a different definition of $g$-twisted $V$-module. Here we prove them starting from the original definition in [H3]. We also prove that when the other conditions for suitable $g$-twisted $V$-modules hold, the duality property for the twisted vertex operators given in [H3] is equivalent to the Jacobi identity for non-logarithmic components of twisted vertex operators given in $[\mathrm{B}]$.

For $\alpha \in \mathbb{C} / \mathbb{Z}$, we denote by

$$
V^{[\alpha]}=\left\{u \in V \mid\left(g-e^{2 \pi \sqrt{-1} \alpha}\right)^{\Lambda} u=0 \text { for some } \Lambda \in \mathbb{Z}_{+}\right\}
$$

the generalized eigenspace with eigenvalue $e^{2 \pi \sqrt{-1} \alpha}$ for $g$. For $\alpha \in \mathbb{C} / \mathbb{Z}$, there is a unique $a \in[0,1)+\mathbb{I}$ such that $a+\mathbb{Z}=\alpha$. We call $a \in[0,1)+\mathbb{I}$ a $g$-weight of $V$ if $V^{[a+\mathbb{Z}]} \neq 0$. We

use $P(V)$ to denote the set of all the $g$-weights of $V$. Then $V=\coprod_{n \in \mathbb{C}, a \in P(V)} V_{(n)}^{[a+\mathbb{Z}]}$. Let $\mathbf{A}_{V}: V \rightarrow V$ be the linear map defined by $\mathbf{A}_{V} v=a v$ for $v \in V^{[a+\mathbb{Z}]}$ and $a \in P(V)$. 
Definition 2.1 A $\overline{\mathbb{C}}_{+}$-graded weak g-twisted $V$-module is a $\overline{\mathbb{C}}_{+} \times \mathbb{C} / \mathbb{Z}$-graded vector space $W=\coprod_{n \in \overline{\mathbb{C}}_{+}, \alpha \in \mathbb{C} / \mathbb{Z}} W_{n}^{[\alpha]}$ (graded by $\overline{\mathbb{C}}_{+}$-degrees and $g$-weights) equipped with a linear map

$$
\begin{gathered}
Y^{g}: V \otimes W \rightarrow W\{x\}[\log x], \\
v \otimes w \mapsto Y^{g}(v, x) w
\end{gathered}
$$

and an action of $g$ satisfying the following conditions:

1. The equivariance property: For $p \in \mathbb{Z}, z \in \mathbb{C}^{\times}, v \in V$ and $w \in W, Y^{g ; p+1}(g v, z) w=$ $Y^{g ; p}(v, z) w$, where for $p \in \mathbb{Z}$,

$$
Y^{g ; p}(v, z) w=\left.Y^{g}(v, x) w\right|_{x^{n}=e^{n l_{p}(z)}, \log x=l_{p}(z)}
$$

is the $p$-th analytic branch of $Y^{g}$.

2. The identity property: For $w \in W, Y^{g}(\mathbf{1}, x) w=w$.

3. The duality property: Let $W^{\prime}=\coprod_{n \in \overline{\mathbb{C}}_{+}, \alpha \in \mathbb{C} / \mathbb{Z}}\left(W_{n}^{[\alpha]}\right)^{*}$ and, for $n \in \mathbb{C}, \pi_{n}: W \rightarrow W_{n}=$ $\coprod_{\alpha \in \mathbb{C} / \mathbb{Z}} W_{n}^{[\alpha]}$ be the projection. For any $u, v \in V, w \in W$ and $w^{\prime} \in W^{\prime}$, there exists a multivalued analytic function of the form

$$
f\left(z_{1}, z_{2}\right)=\sum_{i, j, k, l=0}^{N} a_{i j k l} z_{1}^{m_{i}} z_{2}^{n_{j}}\left(\log z_{1}\right)^{k}\left(\log z_{2}\right)^{l}\left(z_{1}-z_{2}\right)^{-t}
$$

for $N \in \mathbb{N}, m_{1}, \ldots, m_{N}, n_{1}, \ldots, n_{N} \in \mathbb{C}$ and $t \in \mathbb{Z}_{+}$, such that the series

$$
\begin{aligned}
\left\langle w^{\prime}, Y^{g ; p}\left(u, z_{1}\right) Y^{g ; p}\left(v, z_{2}\right) w\right\rangle & =\sum_{n \in \mathbb{C}}\left\langle w^{\prime}, Y^{g ; p}\left(u, z_{1}\right) \pi_{n} Y^{g ; p}\left(v, z_{2}\right) w\right\rangle, \\
\left\langle w^{\prime}, Y^{g ; p}\left(v, z_{2}\right) Y^{g ; p}\left(u, z_{1}\right) w\right\rangle & =\sum_{n \in \mathbb{C}}\left\langle w^{\prime}, Y^{g ; p}\left(v, z_{2}\right) \pi_{n} Y^{g ; p}\left(u, z_{1}\right) w\right\rangle, \\
\left\langle w^{\prime}, Y^{g ; p}\left(Y\left(u, z_{1}-z_{2}\right) v, z_{2}\right) w\right\rangle & =\sum_{n \in \mathbb{C}}\left\langle w^{\prime}, Y^{g ; p}\left(\pi_{n} Y\left(u, z_{1}-z_{2}\right) v, z_{2}\right) w\right\rangle
\end{aligned}
$$

are absolutely convergent in the regions $\left|z_{1}\right|>\left|z_{2}\right|>0,\left|z_{2}\right|>\left|z_{1}\right|>0,\left|z_{2}\right|>\left|z_{1}-z_{2}\right|>$ 0 , respectively, and are convergent to the branch

$$
\sum_{i, j, k, l=0}^{N} a_{i j k l} e^{m_{i} l_{p}\left(z_{1}\right)} e^{n_{j} l_{p}\left(z_{2}\right)} l_{p}\left(z_{1}\right)^{k} l_{p}\left(z_{2}\right)^{l}\left(z_{1}-z_{2}\right)^{-t}
$$

of $f\left(z_{1}, z_{2}\right)$ when $\arg z_{1}$ and $\arg z_{2}$ are sufficiently close (more precisely, when $\mid \arg z_{1}-$ $\left.\arg z_{2} \mid<\frac{\pi}{2}\right)$. 
4. The $\overline{\mathbb{C}}_{+}$- and g-grading conditions: For $v \in V_{(m)}$ and $w \in W_{p}=\coprod_{\alpha \in \mathbb{C} / \mathbb{Z}} W_{p}^{[\alpha]}$ where $m \in$ $\mathbb{Z}$ and $p \in \overline{\mathbb{C}}_{+}$, write $Y^{g}(v, x) w=\sum_{k=0}^{N} \sum_{n \in \mathbb{C}} Y_{n, k}^{g}(v) w x^{-n-1}(\log x)^{k}$. Then $Y_{n, k}^{g}(v) w$ is 0 when $m-n-1+p \notin \overline{\mathbb{C}}_{+}$(or $\Re(m-n-1+p)<0$ ), is in $W_{m-n-1+p}$ when $m-n-1+p \in$ $\overline{\mathbb{C}}_{+}$and for $r \in \mathbb{R}, \coprod_{n \in \mathbb{I}} W_{n}$ is equal to the subspace of $W$ consisting of $w \in W$ such that when $Y_{n, k}^{g}(v) w=0$ for $v \in V_{(m)}, n \in \mathbb{C}, k=0, \ldots, N, m-n-1 \notin \overline{\mathbb{C}}_{+}$. For $\alpha \in \mathbb{C} / \mathbb{Z}, w \in W^{[\alpha]}=\coprod_{n \in \overline{\mathbb{C}}_{+}} W_{n}^{[\alpha]}$, there exists $\Lambda \in \mathbb{Z}_{+}$such that $\left(g-e^{2 \pi \sqrt{-1} \alpha}\right)^{\Lambda} w=0$. Moreover, $g Y^{g}(u, x) v=Y^{g}(g u, x) g v$.

5. The $L(-1)$-derivative property: For $v \in V$,

$$
\frac{d}{d x} Y^{g}(v, x)=Y^{g}(L(-1) v, x)
$$

A lower bounded generalized $g$-twisted $V$-module or simply a lower bounded $g$-twisted $V$ module is a $\overline{\mathbb{C}}_{+}$-graded weak $g$-twisted $V$-module $W$ together with a decomposition of $W$ as a direct sum $W=\bigsqcup_{n \in \mathbb{C}} W_{[n]}$ of generalized eigenspaces $W_{[n]}$ with eigenvalues $n \in \mathbb{C}$ for the operator $L^{g}(0)=\operatorname{Res}_{x} x Y^{g}(\omega, x)$ such that for each $n \in \mathbb{C}$ and each $\alpha \in \mathbb{C} / \mathbb{Z}, W_{[n+l]}^{[\alpha]}=$ $W_{[n+l]} \cap W^{[\alpha]}=0$ for sufficiently negative real number $l$. A lower bounded generalized $g$ twisted $V$-module is said to be strongly $\mathbb{C} / \mathbb{Z}$-graded or grading-restricted if it is lower bounded and for each $n \in \mathbb{C}, \alpha \in \mathbb{C} / \mathbb{Z}, \operatorname{dim} W_{[n]}^{[\alpha]}=\operatorname{dim} W_{[n]} \cap W^{[\alpha]}<\infty$.

In the twisted representation theory of vertex operator algebras, we are mainly interested in strongly $\mathbb{C} / \mathbb{Z}$-graded or grading-restricted generalized $g$-twisted modules. For simplicity, we shall call them simply $g$-twisted $V$-modules. In the case that $g$ does not act on $V$ semisimply, since the twisted vertex operators involve not only powers of the variable but also the logarithm of the variable, we shall also call such a $g$-twisted $V$-module a logarithmic $g$ twisted $V$-module. Lower bounded $g$-twisted $V$-modules also often appear in the formulations of certain conditions and assumptions. $\overline{\mathbb{C}}_{+}$-weak $g$-twisted $V$-modules are not really needed in the twisted representation theory of vertex operator algebras. They are not introduced in [H3 because of this reason. But in this paper, they are needed in the proof of the theorem that $Z_{g}(V)$ and $A_{g}(V)$ are isomorphic. Because of this reason, we prove all the results in this paper for $\overline{\mathbb{C}}_{+}$-weak $g$-twisted $V$-modules and then derive the corresponding results for lower bounded $g$-twisted $V$-modules and $g$-twisted $V$-modules (including logarithmic $g$-twisted $V$-modules) as special cases.

Let $W=\coprod_{n \in \overline{\mathbb{C}}_{+}, \alpha \in \mathbb{C} / \mathbb{Z}} W_{n}^{[\alpha]}$ be a $\overline{\mathbb{C}}_{+}$-graded weak $g$-twisted $V$-module with the twisted vertex operator map $Y^{g}$. For $\alpha \in \mathbb{C} / \mathbb{Z}$, let $W^{[\alpha]}=\coprod_{n \in \overline{\mathbb{C}}_{+}} W_{n}^{[\alpha]}$. When $W$ is a (lower bounded) $g$-twisted $V$-module, we also have $W^{[\alpha]}=\coprod_{n \in \mathbb{C}} W_{[n]}^{[\alpha]}$. As in the case of $V$, we call $a \in[0,1)+\mathbb{I}$ a $g$-weight of $W$ if $W^{[a+\mathbb{Z}]} \neq 0$. Let $P(W)$ be the set of all $g$-weights of $W$. Then $W=\bigsqcup_{n \in \overline{\mathbb{C}}_{+}, a \in P(W)} W_{[n]}^{[a+\mathbb{Z}]}$. Let $\mathbf{A}_{W}: W \rightarrow W$ be the linear map defined by $\mathbf{A}_{W} w=a w$ for $w \in W^{[a+\mathbb{Z}]}$ and $a \in P(W)$. It is clear that for $a \in P(V)$ and $b \in P(W)$, either $a+b \in P(W)$ or $a+b-1 \in P(W)$. 
For $v \in V$, we have

$$
Y^{g}(v, x)=\sum_{k=0}^{N} \sum_{n \in \mathbb{C}} Y_{n, k}^{g}(v) x^{-n-1}(\log x)^{k} .
$$

Let

$$
Y_{k}^{g}(v, x)=\sum_{n \in \mathbb{C}} Y_{n, k}^{g}(v) x^{-n-1} .
$$

Then

$$
Y^{g}(v, x)=\sum_{k=0}^{N} Y_{k}^{g}(v, x)(\log x)^{k} .
$$

Denote the formal variable $\log x$ by $y$ and let

$$
Y^{g}(v, x, y)=\sum_{k=0}^{N} Y_{k}^{g}(v, x) y^{k}=\sum_{k=0}^{N} \sum_{n \in \mathbb{C}} Y_{n, k}^{g}(v) x^{-n-1} y^{k}
$$

for $v \in V$. Taking $u$ in the duality property to be $\mathbf{1}$, we see that $Y_{n, k}^{g}(v) w=0$ for $k=0, \ldots, N$ when $\Re(n)$ is sufficiently negative, that is, $Y_{k}^{g}(v, x) w$ for $k=0, \ldots, N$ are lower truncated. We say that $Y_{k}^{g}, k=0, \ldots, N$, are lower truncated.

Since the homogeneous subspaces of $V$ are finite dimensional and $g$ preserves the homogeneous subspaces of $V$, by the multiplicative Jordan-Chevalley decomposition, there exist a unique semisimple automorphism $\sigma$ of $V$ and a unique locally unipotent operator $g_{u}$ such that $\sigma$ and $g_{u}$ commute with each other and $g=\sigma g_{u}$. Here by locally unipotent operator, we mean that $g_{u}-1_{V}$ is locally nilpotent, that is, for $v \in V,\left(g_{u}-1_{V}\right)^{j} v=0$ when $j$ is sufficiently large. Let

$$
\begin{aligned}
\mathcal{N} & =\frac{\log g_{u}}{2 \pi \sqrt{-1}} \\
& =\frac{\log \left(1_{V}+\left(g_{u}-1_{V}\right)\right)}{2 \pi \sqrt{-1}} \\
& =\sum_{j \in \mathbb{Z}_{+}} \frac{(-1)^{j+1}\left(g_{u}-1_{V}\right)^{j}}{2 \pi \sqrt{-1} j} .
\end{aligned}
$$

Note that since $g_{u}$ is locally unipotent, the right-hand side of (2.2) is a finite sum when acting on $v \in V$ and is locally nilpotent. From the definition of $\mathcal{N}$, we have

$$
\begin{aligned}
g_{u} & =e^{2 \pi \sqrt{-1} \mathcal{N}} \\
& =\sum_{j \in \mathbb{N}} \frac{(2 \pi \sqrt{-1} \mathcal{N})^{j}}{j !} .
\end{aligned}
$$

Thus we have $g=\sigma e^{2 \pi \sqrt{-1} \mathcal{N}}$. 
Let $\left(W, Y^{g}\right)$ be a $\overline{\mathbb{C}}_{+}$-graded weak $g$-twisted $V$-module. We now use the multiplicative Jordan-Chevalley decomposition of $g$ above to derive some formulas. For $n \in \mathbb{Z}$, consider the space $Y^{g}\left(V_{(n)}, x, y\right)$ of elements of (End $\left.W\right)\{x\}[y]$ of the form $Y^{g}(v, x, y)$ for $v \in V_{(n)}$. Then $Y^{g}(\cdot, x, y)$ is a surjective homomorphism from $V_{(n)}$ to $Y^{g}\left(V_{(n)}, x, y\right)$. In particular, $Y^{g}\left(V_{(n)}, x, y\right)$ is finite dimensional. Since $g$ preserve weights, $g$ acts on $V_{(n)}$. Thus $g$ also acts on $Y^{g}\left(V_{(n)}, x, y\right)$ by $g \cdot Y^{g}(v, x, y)=Y^{g}(g v, x, y)$ for $v \in V_{(n)}$. Since on $V_{(n)}, g=\sigma e^{2 \pi \sqrt{-1} \mathcal{N}}$, we obtain a decomposition of this action of $g$ on $Y^{g}\left(V_{(n)}, x, y\right)$ which, for simplicity, we shall still write as $g=\sigma e^{2 \pi \sqrt{-1} \mathcal{N}}$ where $\sigma$ and $\mathcal{N}$ are the corresponding operators on $Y^{g}\left(V_{(n)}, x, y\right)$.

On the other hand, from the equivariance property,

$$
Y^{g}(g v, x, y)=e^{-2 \pi \sqrt{-1} x \partial / \partial x} e^{-2 \pi \sqrt{-1} \partial / \partial y} Y^{g}(v, x, y)
$$

for $v \in V$. By (2.3), the action of $g$ on $Y^{g}\left(V_{(n)}, x, y\right)$ is equal to $e^{-2 \pi \sqrt{-1} x \partial / \partial x} e^{-2 \pi \sqrt{-1} \partial / \partial y}$. If $Y^{g}\left(V_{(n)}, x, y\right)$ is invariant under $e^{-2 \pi \sqrt{-1} x \partial / \partial x}$ and $e^{-2 \pi \sqrt{-1} \partial / \partial y}$, then $e^{-2 \pi \sqrt{-1} x \partial / \partial x} e^{-2 \pi \sqrt{-1} \partial / \partial y}$ is a Jordan-Chevalley decomposition of $g$ on $Y^{g}\left(V_{(n)}, x, y\right)$. By the uniqueness of the JordanChevalley decomposition, we obtain $\sigma=e^{-2 \pi \sqrt{-1} x \partial / \partial x}$ and $e^{2 \pi \sqrt{-1} \mathcal{N}}=e^{-2 \pi \sqrt{-1} \partial / \partial y}$. Since

$$
\begin{aligned}
\partial / \partial y & =\frac{\log \left(1_{Y^{g}\left(V_{(n)}, x, y\right)}+\left(e^{-2 \pi \sqrt{-1} \partial / \partial y}-1_{Y^{g}\left(V_{(n)}, x, y\right)}\right)\right.}{-2 \pi \sqrt{-1}} \\
& =\sum_{j \in \mathbb{Z}_{+}} \frac{(-1)^{j+1}\left(e^{-2 \pi \sqrt{-1} \partial / \partial y}-1_{Y^{g}\left(V_{(n)}, x, y\right)}\right)^{j}}{-2 \pi \sqrt{-1} j}
\end{aligned}
$$

by the definition of $\mathcal{N}$, we obtain $\mathcal{N}=-\partial / \partial y$ on $Y^{g}\left(V_{(n)}, x, y\right)$. Since we do not assume that $Y^{g}\left(V_{(n)}, x, y\right)$ is invariant under $e^{-2 \pi \sqrt{-1} x \partial / \partial x}$ and $e^{-2 \pi \sqrt{-1} \partial / \partial y}$, we have to prove $\sigma=$ $e^{-2 \pi \sqrt{-1} x \partial / \partial x}$ and $e^{2 \pi \sqrt{-1} \mathcal{N}}=e^{-2 \pi \sqrt{-1} \partial / \partial y}$ directly, without using the uniqueness of the JordanChevalley decomposition for an operator on a finite-dimensional space.

Let $v \in V_{(n)}^{[\alpha]}, \alpha \in \mathbb{C} / \mathbb{Z}$. Since the action of $g$ on $Y^{g}\left(V_{(n)}, x, y\right)$ is equal to $e^{-2 \pi \sqrt{-1} x \partial / \partial x} e^{-2 \pi \sqrt{-1} \partial / \partial y}$ and $Y^{g}(v, x, y)$ is a generalized eigenvector of $g$ with eigenvalue $e^{2 \pi \sqrt{-1} \alpha}$, there exists $M \in \mathbb{Z}_{+}$ such that

$$
\left(e^{-2 \pi \sqrt{-1} x \partial / \partial x} e^{-2 \pi \sqrt{-1} \partial / \partial y}-e^{2 \pi \sqrt{-1} \alpha}\right)^{M} Y^{g}(v, x, y)=0 .
$$

From (2.1) and (2.4), we obtain

$$
\begin{aligned}
\sum_{k=0}^{N} & \sum_{m \in \mathbb{C}} Y_{m, k}^{g}(v)\left(e^{-2 \pi \sqrt{-1}(-m-1)} e^{-2 \pi \sqrt{-1} \partial / \partial y}-e^{2 \pi \sqrt{-1} \alpha}\right)^{M} x^{-m-1} y^{k} \\
& =\sum_{k=0}^{N} \sum_{m \in \mathbb{C}} Y_{m, k}^{g}(v)\left(e^{-2 \pi \sqrt{-1} x \partial / \partial x} e^{-2 \pi \sqrt{-1} \partial / \partial y}-e^{2 \pi \sqrt{-1} \alpha}\right)^{M} x^{-m-1} y^{k} \\
& =0 .
\end{aligned}
$$

From (2.5), we see that the coefficients of the left-hand side of (2.5) as a formal series in powers of $x$ must be 0 , that is,

$$
\sum_{k=0}^{N} Y_{m, k}^{g}(v)\left(e^{-2 \pi \sqrt{-1}(-m-1)} e^{-2 \pi \sqrt{-1} \partial / \partial y}-e^{2 \pi \sqrt{-1} \alpha}\right)^{M} y^{k}=0
$$


for $m \in \mathbb{C}$. Rewriting (2.6), we obtain

$$
\left(e^{-2 \pi \sqrt{-1} \partial / \partial y}-e^{2 \pi \sqrt{-1}(\alpha-m-1)}\right)^{M} \sum_{k=0}^{N} Y_{m, k}^{g}(v) y^{k}=0
$$

for $m \in \mathbb{C}$. Let $m \in \mathbb{C}$ such that $e^{2 \pi \sqrt{-1}(\alpha-m-1)} \neq 1$. If $Y_{m, k}^{g}(v) \neq 0$ for some $k$, then $\sum_{k=0}^{N} Y_{m, k}^{g}(v) y^{k}$ is a nonzero polynomial in $y$. Since $e^{2 \pi \sqrt{-1} i(\alpha-m-1)} \neq 1$,

$$
\left(e^{-2 \pi \sqrt{-1} \partial / \partial y}-e^{2 \pi \sqrt{-1}(\alpha-m-1)}\right)^{M} \sum_{k=0}^{N} Y_{m, k}^{g}(v) y^{k}
$$

cannot be equal to 0 . Contradiction with (2.5). Thus for such $m, Y_{m, k}^{g}(v)=0$ for $k=0, \ldots, N$ and we have

$$
Y^{g}(v, x, y)=\sum_{k=0}^{N} \sum_{m \in \alpha} Y_{m, k}^{g}(v) x^{-m-1} y^{k}
$$

In particular,

$$
\begin{aligned}
e^{-2 \pi \sqrt{-1} x \partial / \partial x} Y^{g}(v, x, y) & =e^{2 \pi \sqrt{-1} \alpha} Y^{g}(v, x, y) \\
& =\sigma Y^{g}(v, x, y) .
\end{aligned}
$$

Since $v$ is an arbitrary element of $V_{(n)}, e^{-2 \pi \sqrt{-1} x \partial / \partial x}=\sigma$ on $Y^{g}\left(V_{(n)}, x, y\right)$ and thus $e^{2 \pi \sqrt{-1} \mathcal{N}}=$ $e^{-2 \pi \sqrt{-1} \partial / \partial y}$.

Since $n$ is arbitrary, we have proved the following formulas:

Lemma 2.2 For $v \in V$,

$$
\begin{aligned}
Y^{g}(\sigma v, x, y) & =e^{-2 \pi i x \partial / \partial x} Y^{g}(v, x, y), \\
Y^{g}(\mathcal{N} v, x, y) & =-\partial / \partial y Y^{g}(v, x, y), \\
Y^{g}\left(e^{2 \pi i \mathcal{N}} v, x, y\right) & =e^{-2 \pi i \partial / \partial y} Y^{g}(v, x, y) .
\end{aligned}
$$

From (2.7), (2.8) and (2.9), for $v \in V^{[a+\mathbb{Z}]}, a \in P(V)$ and $w \in W, Y^{g}(v, x) w \in$ $x^{-a} W((x))[\log x]$. Then for $v \in V^{[\alpha]}, w \in W$,

$$
\begin{aligned}
Y_{k}^{g}(v, x) w & =\sum_{n \in a+\mathbb{Z}} Y_{n, k}^{g}(v) w x^{-n-1}, \\
Y^{g}(v, x) w & =\sum_{k=0}^{N_{g}(v)} Y_{k}^{g}(v, x) w(\log x)^{k},
\end{aligned}
$$

where $N_{g}(v) \in \mathbb{N}$ depends only on $\mathcal{N}$ and $v$. For convenience, we shall use $u(n)$ to denote the operator $Y_{n, 0}^{g}(u)$.

From (2.8), we have the following relation between $Y^{g}$ and $Y_{0}^{g}$ : 
Lemma 2.3 For $v \in V$,

$$
Y^{g}(v, x)=Y_{0}^{g}\left(x^{-\mathcal{N}} v, x\right)
$$

where $x^{-\mathcal{N}}=e^{-\mathcal{N} \log x}$.

Proof. From (2.8) and the formal Taylor's theorem, we obtain

$$
\begin{aligned}
Y^{g}\left(x^{\mathcal{N}} v, x, y\right) & =e^{-(\log x) \partial / \partial y} Y^{g}(v, x, y) \\
& =\sum_{k=0}^{N_{g}(v)} e^{-(\log x) \partial / \partial y} Y_{k}^{g}(v, x) y^{k} \\
& =\sum_{k=0}^{N_{g}(v)} Y_{k}^{g}(v, x)(y-\log x)^{k} .
\end{aligned}
$$

Substituting $\log x$ for $y$ in (2.11) and then substituting $x^{-\mathcal{N}} v$ for $v$, we obtain (2.10).

We also need the following lemma:

Lemma 2.4 For $u \in V$, we have

$$
\begin{aligned}
\sigma Y^{g}(u, x) & =Y^{g}(\sigma u, x) \sigma, \\
{\left[\mathcal{N}, Y^{g}(u, x)\right] } & =Y^{g}(\mathcal{N} u, x), \\
e^{y \mathcal{N}} Y^{g}(u, x) & =Y^{g}\left(e^{y \mathcal{N}} u, x\right) e^{y \mathcal{N}} .
\end{aligned}
$$

Proof. Let $u \in V^{[\alpha]}$ and $v \in V^{[\beta]}$. Then $u$ and $v$ are generalized eigenvectors for $g$ with eigenvalues $e^{2 \pi \sqrt{-1} \alpha}$ and $e^{2 \pi \sqrt{-1} \beta}$. In particular, there exist $M, N \in \mathbb{Z}_{+}$such that $(g-$ $\left.e^{2 \pi \sqrt{-1} \alpha}\right)^{M} u=\left(g-e^{2 \pi \sqrt{-1} \beta}\right)^{N} v=0$.

Using the fact that $g$ is an automorphism of $V$, we have

$$
\left(g-e^{2 \pi \sqrt{-1}(\alpha+\beta)}\right) Y^{g}(u, x) v=Y^{g}\left(\left(g-e^{2 \pi \sqrt{-1} \alpha}\right) u, x\right) g v+Y^{g}\left(e^{2 \pi \sqrt{-1} \alpha} u, x\right)\left(g-e^{2 \pi \sqrt{-1} \beta}\right) v .
$$

Then

$$
\begin{aligned}
& \left(g-e^{2 \pi \sqrt{-1}(\alpha+\beta)}\right)^{M+N} Y^{g}(u, x) v \\
& \quad=\sum_{j=0}^{M+N}\left(\begin{array}{c}
M+N \\
j
\end{array}\right) Y^{g}\left(e^{2 \pi \sqrt{-1}(M+N-j) \alpha}\left(g-e^{2 \pi \sqrt{-1} \alpha}\right)^{j} u, x\right) g^{j}\left(g-e^{2 \pi \sqrt{-1} \beta}\right)^{M+N-j} v \\
& \quad=0 .
\end{aligned}
$$

Thus $Y^{g}(u, x) v \in V^{[\alpha+\beta]}\left[\left[x, x^{-1}\right]\right]$. This proves (2.12) .

Using (2.12) together with the fact that $g$ is an automorphism of $V$, we obtain

$$
e^{2 \pi \sqrt{-1} \mathcal{N}} Y^{g}(u, x)=Y^{g}\left(e^{2 \pi \sqrt{-1} \mathcal{N}} u, x\right) e^{2 \pi \sqrt{-1} \mathcal{N}}
$$


or

$$
\begin{aligned}
Y^{g}\left(e^{2 \pi \sqrt{-1} \mathcal{N}} u, x\right) & =e^{2 \pi \sqrt{-1} \mathcal{N}} Y^{g}(u, x) e^{-2 \pi \sqrt{-1} \mathcal{N}} \\
& =\operatorname{Ad}\left(e^{2 \pi \sqrt{-1} \mathcal{N}}\right)\left(Y^{g}(u, x)\right)
\end{aligned}
$$

Thus by the definition of $\mathcal{N}$,

$$
\begin{aligned}
Y^{g}(\mathcal{N} u, x) & =\frac{1}{2 \pi \sqrt{-1}} Y^{g}\left(\log \left(e^{2 \pi \sqrt{-1} \mathcal{N}}\right) u, x\right) \\
& \left.=\frac{1}{2 \pi \sqrt{-1}}\left(\log \left(\operatorname{Ad}\left(e^{2 \pi \sqrt{-1} \mathcal{N}}\right)\right)\right)\right)\left(Y^{g}(u, x)\right) \\
& =\frac{1}{2 \pi \sqrt{-1}} \operatorname{ad}(2 \pi \sqrt{-1} \mathcal{N})\left(Y^{g}(u, x)\right) \\
& =\left[\mathcal{N}, Y^{g}(u, x)\right]
\end{aligned}
$$

proving (2.13). The formula (2.14) follows immediately from (2.13).

As consequences of Lemmas 2.3 and 2.4, we have the following properties:

Proposition 2.5 For $n \in \mathbb{Z}$ and $v \in V$,

$$
\begin{aligned}
{[\mathcal{N}, L(n)] } & =0 \\
x^{\mathcal{N}} L(n) & =L(n) x^{\mathcal{N}}, \\
Y_{0}^{g}(L(-1) u, x) & =\frac{d}{d x} Y_{0}^{g}(u, x)-x^{-1} Y_{0}^{g}(\mathcal{N} u, x) .
\end{aligned}
$$

Proof. Since $g \omega=\omega$, we have $\mathcal{N} \omega=\omega$. Then (2.16) follows from (2.13) and (2.17) is an immediate consequence of (2.16) $)$.

The formula (2.18) is obtained by taking derivative with respect to $x$ on both sides of (2.10), using the $L(-1)$-derivative property for $Y^{g}$ and then take the 0 -th power of $\log x$ on both sides of the resulting equality.

Using the the formulas obtained above, we prove a duality property for the map $Y_{0}^{g}$. For $u \in V$, in the region $\left|z_{2}\right|>\left|z_{1}-z_{2}\right|>0$, we use

$$
\left(1+\frac{z_{1}-z_{2}}{z_{2}}\right)^{\mathcal{N}} u
$$

to denote the power series in the variable $\frac{z_{1}-z_{2}}{z_{2}}$ with coefficients in $V$ obtained by using the binomial expansion. This series can also be obtained by writing (2.19) as

$$
\exp \left(\log \left(1+\frac{z_{1}-z_{2}}{z_{2}}\right) \mathcal{N}\right) u
$$


expanding (2.20) as a power series in

$$
\log \left(1+\frac{z_{1}-z_{2}}{z_{2}}\right)
$$

and then expanding powers of (2.21) as powers series in $\frac{z_{1}-z_{2}}{z_{2}}$ in the region $\left|z_{2}\right|>\left|z_{1}-z_{2}\right|>0$. Since $\mathcal{N}$ is locally nilpotent, this series is in fact obtained from a polynomial in (2.21) with coefficients in $V$ by expanding powers of (2.21) as powers series in $\frac{z_{1}-z_{2}}{z_{2}}$ in $\left|z_{2}\right|>\left|z_{1}-z_{2}\right|>0$. In other words, (2.19) is a finite sum of elements of $V$ multiplied by powers of (2.21), which as power series in $\frac{z_{1}-z_{2}}{z_{2}}$ are absolutely convergent in the region $\left|z_{2}\right|>\left|z_{1}-z_{2}\right|>0$.

Theorem 2.6 Let $W$ be a $\overline{\mathbb{C}}_{+}$-graded weak g-twisted $V$-module. Then for $u \in V^{[a+\mathbb{Z}]}, v \in$ $V^{[b+\mathbb{Z}]}, a, b \in P(V)$,

$$
\begin{gathered}
\left\langle w^{\prime}, Y_{0}^{g}\left(u, z_{1}\right) Y_{0}^{g}\left(v, z_{2}\right) w\right\rangle \\
\left\langle w^{\prime}, Y_{0}^{g}\left(v, z_{2}\right) Y_{0}^{g}\left(u, z_{1}\right) w\right\rangle \\
\left\langle w^{\prime}, Y_{0}^{g}\left(Y\left(\left(1+\frac{z_{1}-z_{2}}{z_{2}}\right)^{\mathcal{N}} u, z_{1}-z_{2}\right) v, z_{2}\right) w\right\rangle
\end{gathered}
$$

are absolutely convergent in the region $\left|z_{1}\right|>\left|z_{2}\right|>0,\left|z_{2}\right|>\left|z_{1}\right|>0,\left|z_{2}\right|>\left|z_{1}-z_{2}\right|>0$, respectively, to a function of $z_{1}$ and $z_{2}$ of the form

$$
e^{-a \log z_{1}} e^{-b \log z_{2}} \frac{g\left(z_{1}, z_{2}\right)}{z_{1}^{r} z_{2}^{s}\left(z_{1}-z_{2}\right)^{t}},
$$

where $g\left(z_{1}, z_{2}\right)$ is a polynomial in $z_{1}$ and $z_{2}, r, s, t \in \mathbb{Z}$.

Proof. Since $u \in V^{[a+\mathbb{Z}]}, v \in V^{[b+\mathbb{Z}]}, a, b \in P(V)$, by the duality property,

$$
\begin{gathered}
\left\langle w^{\prime}, Y^{g}\left(u, z_{1}\right) Y^{g}\left(v, z_{2}\right) w\right\rangle, \\
\left\langle w^{\prime}, Y^{g}\left(v, x_{2}\right) Y^{g}\left(u, x_{1}\right) w\right\rangle, \\
\left\langle w^{\prime}, Y^{g}\left(Y\left(u, z_{1}-z_{2}\right) v, z_{2}\right) w\right\rangle
\end{gathered}
$$

are absolutely convergent in the region $\left|z_{1}\right|>\left|z_{2}\right|>0,\left|z_{2}\right|>\left|z_{1}\right|>0,\left|z_{2}\right|>\left|z_{1}-z_{2}\right|>0$, respectively, and are convegent when $\left|\arg z_{1}-\arg z_{2}\right|<\frac{\pi}{2}$ to a function of $z_{1}$ and $z_{2}$ of the form

$$
\begin{gathered}
\sum_{i, j, k, l=0}^{N} a_{i j k l} e^{\left(-a-r_{i}\right) \log z_{1}} e^{\left(-b-s_{j}\right) \log z_{2}}\left(\log z_{1}\right)^{k}\left(\log z_{2}\right)^{l}\left(z_{1}-z_{2}\right)^{-t} \\
=e^{-a \log z_{1}} e^{-b \log z_{2}} \sum_{k, l=0}^{N} \frac{g_{k l}\left(z_{1}, z_{2}\right)}{z_{1}^{r_{0}} z_{2}^{s_{0}}\left(z_{1}-z_{2}\right)^{t}}\left(\log z_{1}\right)^{k}\left(\log z_{2}\right)^{l}
\end{gathered}
$$


where $r_{i}, s_{j}, t \in \mathbb{Z}$ satisfying $r_{i}<r_{i-1}$ and $s_{j}<s_{j-1}$ and $g_{k l}\left(z_{1}, z_{2}\right)$ are polynomials in $z_{1}$ and $z_{2}$. In particular, in the region $\left|z_{1}\right|>\left|z_{2}\right|>0$,

$$
\begin{aligned}
& \sum_{k, l=0}^{N}\left\langle w^{\prime}, Y_{k}^{g}\left(u, z_{1}\right) Y_{l}^{g}\left(v, z_{2}\right) w\right\rangle\left(\log z_{1}\right)^{k}\left(\log z_{2}\right)^{l} \\
& \quad=\left\langle w^{\prime}, Y^{g}\left(u, z_{1}\right) Y^{g}\left(v, z_{2}\right) w\right\rangle \\
& \quad=e^{-a \log z_{1}} e^{-b \log z_{2}} \sum_{k, l=0}^{N} \frac{g_{k l}\left(z_{1}, z_{2}\right)}{z_{1}^{r_{0}} z_{2}^{s_{0}}\left(z_{1}-z_{2}\right)^{t}}\left(\log z_{1}\right)^{k}\left(\log z_{2}\right)^{l} .
\end{aligned}
$$

By Proposition 7.8 in [HLZ], we obtain

$$
\left\langle w^{\prime}, Y_{k}^{g}\left(u, z_{1}\right) Y_{l}^{g}\left(v, z_{2}\right) w\right\rangle=e^{-a \log z_{1}} e^{-b \log z_{2}} \frac{g_{00}\left(z_{1}, z_{2}\right)}{z_{1}^{r_{0}} z_{2}^{s_{0}}\left(z_{1}-z_{2}\right)^{t}} .
$$

Taking $g\left(z_{1}, z_{2}\right)$ to be $g_{00}\left(z_{1}, z_{2}\right)$ and $r, s$ to be $r_{0}, s_{0}$, we see that (2.22) indeed converges absolutely to a function of the form (2.25).

Similarly we can prove that (2.23) converges absolutely to a function of the form (2.25). Since (2.26) and (2.27) converge to the same function in $\left|z_{1}\right|>\left|z_{2}\right|>0$ and $\left|z_{2}\right|>\left|z_{1}\right|>$ 0 , respectively, from the argument above, the fact that the powers of $z_{1}$ and $z_{2}$ in these expressions are congruent to $a$ and $b$, respectively, and Proposition 7.8 in [HLZ], we see that (2.22) and (2.23) converge absolutely to the same function of the form (2.25) in $\left|z_{1}\right|>\left|z_{2}\right|>0$ and $\left|z_{2}\right|>\left|z_{1}\right|>0$, respectively.

The same argument above shows that

$$
\left\langle w^{\prime}, Y_{0}^{g}\left(Y\left(u, z_{1}-z_{2}\right) v, z_{2}\right) w\right\rangle
$$

converges absolutely in the region $\left|z_{2}\right|>\left|z_{1}-z_{2}\right|>0$. As is discussed above, (2.19) is a finite sum of elements of $V$ multiplied by powers of (2.21), which as power series in $\frac{z_{1}-z_{2}}{z_{2}}$ are absolutely convergent in the region $\left|z_{2}\right|>\left|z_{1}-z_{2}\right|>0$, (2.24) is absolutely convergent in the same region. We shall use the associativity for $Y^{g}$ and (2.10) to show that (2.24) converges to the same function that (2.22) converges to.

By (2.10), (2.14) and the associativity for $Y^{g}$, in the region $\left|z_{1}\right|>\left|z_{2}\right|>\left|z_{1}-z_{2}\right|>0$, we have

$$
\begin{aligned}
\left\langle w^{\prime}\right. & \left., Y_{0}^{g}\left(Y\left(\left(1+\frac{z_{1}-z_{2}}{z_{2}}\right)^{\mathcal{N}} u, z_{1}-z_{2}\right) v, z_{2}\right) w\right\rangle \\
& =\left\langle w^{\prime}, Y^{g}\left(z_{2}^{\mathcal{N}} Y\left(\left(\frac{z_{1}}{z_{2}}\right)^{\mathcal{N}} u, z_{1}-z_{2}\right) v, z_{2}\right) w\right\rangle \\
& =\left\langle w^{\prime}, Y^{g}\left(Y\left(z_{1}^{\mathcal{N}} u, z_{1}-z_{2}\right) z_{2}^{\mathcal{N}} v, z_{2}\right) w\right\rangle \\
& =\left\langle w^{\prime}, Y^{g}\left(\left(z_{1}\right)^{\mathcal{N}} u, z_{1}\right) Y^{g}\left(z_{2}^{\mathcal{N}} v, z_{2}\right) w\right\rangle \\
& =\left\langle w^{\prime}, Y_{0}^{g}\left(u, z_{1}\right) Y_{0}^{g}\left(v, z_{2}\right) w\right\rangle .
\end{aligned}
$$


In [B], Bakalov derived a Jacobi identity for the map $Y_{0}^{g}$ from an associator formula for twisted $V$-modules. From Theorem 2.6, we obtain the Jacobi identity immediately.

Theorem 2.7 (Bakalov $[\mathbf{B}]$ ) Let $W$ be a $\overline{\mathbb{C}}_{+}$-graded weak g-twisted $V$-module. Then we have the twisted Jacobi identity

$$
\begin{array}{r}
x_{0}^{-1} \delta\left(\frac{x_{1}-x_{2}}{x_{0}}\right) Y_{0}^{g}\left(u, x_{1}\right) Y_{0}^{g}\left(v, x_{2}\right)-x_{0}^{-1} \delta\left(\frac{-x_{2}+x_{1}}{x_{0}}\right) Y_{0}^{g}\left(v, x_{2}\right) Y_{0}^{g}\left(u, x_{1}\right) \\
=x_{1}^{-1} \delta\left(\frac{x_{2}+x_{0}}{x_{1}}\right)\left(\frac{x_{2}+x_{0}}{x_{1}}\right)^{a} Y_{0}^{g}\left(Y\left(\left(1+\frac{x_{0}}{x_{2}}\right)^{\mathcal{N}} u, x_{0}\right) v, x_{2}\right)
\end{array}
$$

or equivalently, the component form of the twisted Jacobi identity

$$
\begin{aligned}
\sum_{k \in \mathbb{N}}(-1)^{k}\left(\begin{array}{l}
l \\
k
\end{array}\right) u(l- & k+m) v(k+n)-\sum_{k \in \mathbb{N}}(-1)^{k-l}\left(\begin{array}{l}
l \\
k
\end{array}\right) v(l-k+n) u(k+m) \\
& =\sum_{j \in \mathbb{N}}\left(\left(\left(\begin{array}{c}
l+\mathcal{N} \\
j
\end{array}\right) u\right)_{j} v\right)(m+n-j)
\end{aligned}
$$

for $u \in V^{[a+\mathbb{Z}]}, v \in V^{[b+\mathbb{Z}]}, m \in a+\mathbb{Z}, n \in b+\mathbb{Z}$ and $l \in \mathbb{Z}$, where $u(p)=Y_{p, 0}^{g}(u)$ and $v(q)=Y_{q, 0}^{g}(v)$ for $p \in a+\mathbb{Z}$ and $q \in b+\mathbb{Z}$.

As a consequence of Jacobi identity, we have the commutator formulas:

Corollary 2.8 For $u \in V^{[a+\mathbb{Z}]}, v \in V^{[b+\mathbb{Z}]}, m \in a+\mathbb{Z}$ and $n \in b+\mathbb{Z}$, we have

$$
\begin{aligned}
{\left[Y_{0}^{g}\left(u, x_{1}\right), Y_{0}^{g}\left(v, x_{2}\right)\right]=} & \operatorname{Res}_{x_{0}} x_{1}^{-1} \delta\left(\frac{x_{2}+x_{0}}{x_{1}}\right)\left(\frac{x_{2}+x_{0}}{x_{1}}\right)^{a} . \\
& \cdot Y_{0}^{g}\left(Y\left(\left(1+\frac{x_{0}}{x_{2}}\right)^{\mathcal{N}} u, x_{0}\right) v, x_{2}\right), \\
{\left[u(m), Y_{0}^{g}\left(v, x_{2}\right)\right]=} & \sum_{j=0}^{\infty} x_{2}^{m-j} Y_{0}^{g}\left(\left(\left(\begin{array}{c}
m+\mathcal{N} \\
j
\end{array}\right) u\right)(j) v, x_{2}\right), \\
{[u(m), v(n)]=} & \sum_{j=0}^{\infty}\left(\left(\left(\begin{array}{c}
m+\mathcal{N} \\
j
\end{array}\right) u\right)_{j} v\right)(m+n-j) .
\end{aligned}
$$

We also have the associator formula and weak associativity:

Corollary 2.9 For $u \in V^{[a+\mathbb{Z}]}, v \in V$ and $w \in W$,

$$
\begin{gathered}
\operatorname{Res}_{x_{1}} x_{1}^{a} x_{0}^{-1} \delta\left(\frac{x_{1}-x_{2}}{x_{0}}\right) Y_{0}^{g}\left(u, x_{1}\right) Y_{0}^{g}\left(v, x_{2}\right)-\operatorname{Res}_{x_{1}} x_{1}^{a} x_{0}^{-1} \delta\left(\frac{-x_{2}+x_{1}}{x_{0}}\right) Y_{0}^{g}\left(v, x_{2}\right) Y_{0}^{g}\left(u, x_{1}\right) \\
=\left(x_{2}+x_{0}\right)^{a} Y_{0}^{g}\left(Y\left(\left(1+\frac{x_{0}}{x_{2}}\right)^{\mathcal{N}} u, x_{0}\right) v, x_{2}\right)
\end{gathered}
$$


For $u \in V^{[a+\mathbb{Z}]}, v \in V$ and $w \in W$, let $l \in a+\mathbb{Z}$ such that $u(n) w=0$ for $n \geq l$. Then

$$
\left(x_{0}+x_{2}\right)^{l} Y_{0}^{g}\left(u, x_{0}+x_{2}\right) Y_{0}^{g}\left(v, x_{2}\right) w=\left(x_{2}+x_{0}\right)^{l} Y_{0}^{g}\left(Y\left(\left(1+\frac{x_{0}}{x_{2}}\right)^{\mathcal{N}} u, x_{0}\right) v, x_{2}\right) w .
$$

Finally we have the following equivalence of the main properties of $\overline{\mathbb{C}}_{+}$-graded weak $g$-twisted modules:

Theorem 2.10 The following properties for a $\overline{\mathbb{C}}_{+}$-graded weak $g$-twisted $V$-module are equivalent:

(1) The duality property for $Y^{g}$ in Definition 2.1.

(2) The duality property for $Y_{0}^{g}$ in Theorem [2.6.

(3) The property that $Y_{0}^{g}$ is lower truncated and the twisted Jacobi identity (2.29).

(4) The property that $Y_{0}^{g}$ is lower truncated, the commutator formula (2.31) and the weak associativity (2.35).

Proof. We have shown that $(1) \Rightarrow(2) \Rightarrow(3) \Rightarrow(4)$. The same method as that for ordinary modules shows that $(4) \Rightarrow(2)$. Using (2.10), we see that $(2) \Rightarrow(1)$.

\section{The $g$-twisted universal enveloping algebra and $g$ - twisted zero-mode algebra}

In this section, we construct two associative algebras associated with $V$ and the automorphism $g$, one is called the " $g$-twisted universal algebra of $V$ " and the other is called the " $g$-twisted zero-mode algebra associated to $V "$.

Let

$$
L(V, g)=\coprod_{a \in P(V)} V^{[a+\mathbb{Z}]} \otimes t^{a} \mathbb{C}\left[t, t^{-1}\right]
$$

For $u \in V^{[a+\mathbb{Z}]}$ and $m \in a+\mathbb{Z}$, we use $u(m)$ to denote the element $u \otimes t^{m} \in L(V, g)$.

Let $T(L(V, g))$ be the tensor algebra of $L(V, g)$. Then $T(L(V, g))$ is spanned by elements of the form

$$
u_{1}\left(i_{1}\right) \otimes \cdots \otimes u_{n}\left(i_{n}\right)
$$

for $u_{s} \in V^{\left[a_{s}+\mathbb{Z}\right]}, i_{s} \in a_{s}+\mathbb{Z}$. For simplicity, we omit the tensor symbols for the elements. Then the multiplication is given by

$$
\left(u_{1}\left(i_{1}\right) \cdots u_{m}\left(i_{m}\right)\right)\left(v_{1}\left(j_{1}\right) \cdots v_{n}\left(j_{n}\right)\right)=u_{1}\left(i_{1}\right) \cdots u_{m}\left(i_{m}\right) v_{1}\left(j_{1}\right) \cdots v_{n}\left(j_{n}\right) .
$$


Define a grading called weight on $T(L(V, g))$ as follows: For $\lambda \in \mathbb{C}$,

$$
\text { wt } \lambda=0
$$

and for $\lambda \in \mathbb{C}$, homogeneous $u_{s} \in V^{\left[a_{s}+\mathbb{Z}\right]}$ and $i_{s} \in a_{s}+\mathbb{Z}$,

$$
\text { wt }\left(\lambda u_{1}\left(i_{1}\right) \cdots u_{n}\left(i_{n}\right)\right)=\sum_{s=1}^{n}\left(\text { wt } u_{s}-i_{s}-1\right) .
$$

Let $\tilde{P}(V)$ be the subset of $[0,1)+\mathbb{I}$ such that $\tilde{P}(V)+\mathbb{Z}$ is the subgroup of $\mathbb{C} / \mathbb{Z}$ generated by $P(V)+\mathbb{Z}$. Then the weight of a homogeneous element of $T(L(V, g))$ is in $\tilde{P}(V)+\mathbb{Z}$. Let $T(L(V, g))_{k}$ denote the subspace of $T(L(V, g))$ consisting of the elements of weight $k$. Then we have

$$
T(L(V, g))=\coprod_{k \in \tilde{P}(V)+\mathbb{Z}} T(L(V, g))_{k}
$$

and

$$
T(L(V, g))_{k} T(L(V, g))_{l} \subset T(L(V, g))_{k+l} .
$$

For $k \in \tilde{P}(V)+\mathbb{Z}$ and $n \in \mathbb{Z}$, let

$$
T(L(V, g))_{k}^{n}=\coprod_{a \in \tilde{P}(V), i \in n+\mathbb{N}} T(L(V, g))_{k+a+i} T(L(V, g))_{-a-i} .
$$

Then we have

$$
T(L(V, g))_{k}^{n+1} \subset T(L(V, g))_{k}^{n}
$$

and also

$$
\begin{aligned}
& \bigcap_{n \in \mathbb{Z}} T(L(V, g))_{k}^{n}=0, \\
& \bigcup_{n \in \mathbb{Z}} T(L(V, g))_{k}^{n}=T(L(V, g))_{k} .
\end{aligned}
$$

Hence, $\left\{T(L(V, g))_{k}^{n} \mid n \in \mathbb{Z}\right\}$ is a fundamental neighborhood system of $T(L(V, g))_{k}$. Denote by $\tilde{T}(L(V, g))_{k}$ its completion and let

$$
\tilde{T}(L(V, g))=\coprod_{k \in \tilde{P}(V)+\mathbb{Z}} \tilde{T}(L(V, g))_{k} .
$$

Then $\tilde{T}(L(V, g))$ is a complete topological vector space.

Proposition 3.1 The multiplication for $T(L(V, g))$ is continuous under the topology given by the fundamental neighborhood system above. In particular, its topological completion $\tilde{T}(L(V, g))$ is a complete topological ring. 
Proof. It suffices to prove that given $m \in \tilde{P}(V)+\mathbb{Z}$ and $n \in \mathbb{Z}$ and given $k, l \in \tilde{P}(V)+\mathbb{Z}$ satisfying $k+l=m$, there exist $n_{1}, n_{2} \in \mathbb{Z}$ such that

$$
T(L(V, g))_{k}^{n_{1}} T(L(V, g))_{l}+T(L(V, g))_{k} T(L(V, g))_{l}^{n_{2}} \subset T(L(V, g))_{m}^{n} .
$$

Let $l=c+l^{\prime}$ where $c \in \tilde{P}(V)$ and $l^{\prime} \in \mathbb{Z}$. We take $n_{1}=n+l^{\prime}+1$ and $n_{2}=n$. Elements of $T(L(V, g))_{k}^{n+l^{\prime}}$ are finite sums of elements of the form $\varphi \psi$ where $\varphi \in T(L(V, g))_{k+a+i}$ and $\psi \in T(L(V, g))_{-a-i}$ for $a \in \tilde{P}(V), i \in n+l^{\prime}+1+\mathbb{N}$. Let $\eta \in T(L(V, g))_{l}$. When $a-c \in \tilde{P}(V)$, $\varphi \in T(L(V, g))_{(k+l)+(a-c)+\left(i-l^{\prime}\right)}$ and $\psi \eta \in T(L(V, g))_{-(a-c)-\left(i-l^{\prime}\right)}$. Since $a-c \in \tilde{P}(V)$ and $i-l^{\prime} \in n+1+\mathbb{N} \subset n+\mathbb{N}$,

$$
\begin{aligned}
\varphi \psi \eta & \in T(L(V, g))_{(k+l)+(a-c)+\left(i-l^{\prime}\right)} T(L(V, g))_{-(a-c)-\left(i-l^{\prime}\right)} \\
& \subset T(L(V, g))_{k+l}^{n} \\
& =T(L(V, g))_{m}^{n} .
\end{aligned}
$$

When $a-c \notin \tilde{P}(V)$, we must have $a-c+1 \in \tilde{P}(V)$. Then $\varphi \in T(L(V, g))_{(k+l)+(a-c+1)+\left(i-l^{\prime}-1\right)}$ and $\psi \eta \in T(L(V, g))_{-(a-c+1)-\left(i-l^{\prime}-1\right)}$. Since $a-c+1 \in \tilde{P}(V)$ and $i-l^{\prime}-1 \in n+\mathbb{N}$,

$$
\begin{aligned}
\varphi \psi \eta & \in T(L(V, g))_{(k+l)+(a-c+1)+\left(i-l^{\prime}-1\right)} T(L(V, g))_{-(a-c+1)-\left(i-l^{\prime}-1\right)} \\
& \subset T(L(V, g))_{k+l}^{n} \\
& =T(L(V, g))_{m}^{n} .
\end{aligned}
$$

Since the addition is continuous with respect to the topology and elements of $T(L(V, g))_{k}^{n+l^{\prime}}$ are finite sums of elements of the form $\varphi \psi$ above, we have shown that

$$
T(L(V, g))_{k}^{n_{1}} T(L(V, g))_{l} \subset T(L(V, g))_{m}^{n} .
$$

Similarly, elements of $T(L(V, g))_{l}^{n}$ are finite sums of elements of the form $\varphi \psi$ where $\varphi \in T(L(V, g))_{l+a+i}$ and $\psi \in T(L(V, g))_{-\alpha-i}$ for $a \in \tilde{P}(V), i \in n+\mathbb{N}$. Let $\eta \in T(L(V, g))_{k}$. Then $\eta \varphi \in T(L(V, g))_{k+l+a+i}$ and

$$
\begin{aligned}
\eta \varphi \psi & \in T(L(V, g))_{k+l+a+i} T(L(V, g))_{-a-i} \\
& \subset T(L(V, g))_{k+l}^{n} \\
& =T(L(V, g))_{m}^{n} .
\end{aligned}
$$

Thus

$$
T(L(V, g))_{k} T(L(V, g))_{l}^{n_{2}} \subset T(L(V, g))_{m}^{n} .
$$

Combining (3.2) and (3.3), we obtain (3.1).

Proposition 3.2 For $k \in \tilde{P}(V)+\mathbb{Z}$, elements of $\tilde{T}(L(V, g))_{k}$ are of the form $u_{1}+u_{2}$ where $u_{1} \in T(L(V, g))_{k}$ and $u_{2}$ is in the topological completion of

$$
\coprod_{a \in \tilde{P}(V), i \in \mathbb{Z}_{+}} T(L(V, g))_{k+a+i} T(L(V, g))_{-a-i} .
$$


Proof. The topological completion $\tilde{T}(L(V, g))_{k}$ of $T(L(V, g))_{k}$ is the space of all Cauchy sequences or equivalently all Cauchy series in $T(L(V, g))_{k}$. Assuming that $\sum_{m \in \mathbb{Z}_{+}} w_{m}$ where $w_{m} \in T(L(V, g))_{k}$ is such a Cauchy series. Then for any $n \in \mathbb{Z}$,

$$
\sum_{m=M}^{M+N} w_{m} \in \coprod_{a \in \tilde{P}(V), i \in n+\mathbb{N}} T(L(V, g))_{k+a+i} T(L(V, g))_{-a-i}
$$

for sufficiently large $M \in \mathbb{Z}_{+}$and $N \in \mathbb{Z}_{+}$. In particular, if we take $n=1$, then there exists $M \in \mathbb{Z}_{+}$such that $w_{m} \in \coprod_{a \in \tilde{P}(V), i \in \mathbb{Z}_{+}} T(L(V, g))_{k+a+i} T(L(V, g))_{-a-i}$ for $m>M$. Let $u_{1}=\sum_{m=1}^{M} w_{m}$ and $u_{2}=\sum_{m=M+1}^{\infty} w_{m}$. Then $u_{1}$ as a finite sum of elements of $T(L(V, g))_{k}$ is still an element of $T(L(V, g))_{k}$. Since $\sum_{m \in \mathbb{Z}_{+}} w_{m}$ is a Cauchy series, $u_{2}=\sum_{m=M+1}^{\infty} w_{m}$ is also a Cauchy series. Since $w_{m} \in \coprod_{a \in \tilde{P}(V), i \in \mathbb{Z}_{+}} T(L(V, g))_{k+a+i} T(L(V, g))_{-a-i}$ when $m>M$, it is in fact a Cauchy series in $\coprod_{a \in \tilde{P}(V), i \in \mathbb{Z}_{+}} T(L(V, g))_{k+a+i} T(L(V, g))_{-a-i}$ and thus is in its topological completion.

Note that the two terms in the left-hand side of (2.30) and the right-hand side of (2.30) both correspond to well defined elements of $\tilde{T}(L(V, g))$. Taking the difference between the sum of the two elements of $\tilde{T}(L(V, g))$ corresponding to the two terms in the left-hand side of (2.30) and the element of $\tilde{T}(L(V, g))$ corresponding to the right-hand side of (2.30), we obtain an element of $\tilde{T}(L(V, g))$. These are the elements of $\tilde{T}(L(V, g))$ corresponding to the Jacobi identity (2.30). Let $J$ be the two-sided ideal in $\tilde{T}(L(V, g))$ generated by these elements of $\tilde{T}(L(V, g))$ corresponding to the Jacobi identity (2.30), the elements $\mathbf{1}(n)-\delta_{n,-1}$ for $n \in \mathbb{Z}$ corresponding to the identity property for $Y_{0}^{g}$, and the elements

$$
(L(-1) u)(n)+(n+\mathcal{N}) u(n-1)
$$

for $n \in \mathbb{Z}$ and $u \in V$ corresponding to the $L(-1)$-derivative property (2.18).

Definition 3.3 The $g$-twisted universal enveloping algebra $U_{g}(V)$ of $V$ is the quotient algebra $\tilde{T}(L(V, g)) / J$ of $\tilde{T}(L(V, g))$ by the two-sided ideal $J$. Since $J$ is generated by homogeneous elements, the grading on $\tilde{T}(L(V, g))$ induces a grading on $U_{g}(V)$ and we still call the degree of an element of $U_{g}(V)$ its weight. The homogeneous subspace of $U_{g}(V)$ of weight $n \in \mathbb{C}$ is denoted by $U_{g}(V)_{n}$.

In the special case that $g=1_{V}$, the $g$-twisted universal enveloping algebra $U_{g}(V)$ of $V$ is the universal enveloping algebra $U(V)$ of $V$ introduced by Frenkel and Zhu [FZ].

The action of $g$ on $V^{[\alpha]}$ for $\alpha \in \mathbb{C} / \mathbb{Z}$ induces an action of $g$ on $L(V, g)$. This action of $g$ on $L(V, g)$ induces an action of $g$ on $T(L(V, g))$ such that this action in fact gives an automorphism of the associative algebra $T(L(V, g))$. By the definition of the topological completion of $T(L(V, g))$, this action of $g$ on $T(L(V, g))$ extends continuously to an automorphism of the topological completion $\tilde{T}(L(V, g))$. Since the elements of $\tilde{T}(L(V, g))$ corresponding to (2.30), the elements $\mathbf{1}(n)-\delta_{n,-1}$ and (3.4) for $n \in \mathbb{Z}$ and $u$ in generalized eigenspaces for $g$ are also in generalized eigenspaces for $g$ in $\tilde{T}(L(V, g)), U_{g}(V)$ as the quotient of $\tilde{T}(L(V, g))$ 
by the ideal generated by these elements also has an action of $g$. Moreover, the action of $g$ on $U_{g}(V)$ is an automorphism of $U_{g}(V)$.

The following result follows immediately from Proposition 3.2 .

Proposition 3.4 For $k \in \mathbb{C}$, let $\pi_{U_{g}(V)_{k}}$ be the projections from $\tilde{T}(L(V, g))_{k}$ to $U_{g}(V)_{k}$. Then elements of $U_{g}(V)_{k}$ are of the form $u_{1}+u_{2}$ where $u_{1} \in \pi_{U_{g}(V)} T(L(V, g))_{k}$, and $u_{2}$ is in the topological completion of

$$
\coprod_{a \in \tilde{P}(V), i \in \mathbb{Z}_{+}}\left(\pi_{U_{g}(V)} T(L(V, g))_{k+a+i}\right)\left(\pi_{U_{g}(V)} T(L(V, g))_{-a-i}\right) .
$$

Proposition 3.5 Let $W$ be a $\overline{\mathbb{C}}_{+}$-graded weak $V$-module. Then $W$ has a natural structure of a $U_{g}(V)$-module such that the $U_{g}(V)$-module structure is compatible with the action of $g$ on $U_{g}(V)$ and $W$ in the sense that for $u \in U_{g}(V)$ and $w \in W, g(u w)=(g u)(g w)$. Conversely, let $W=\coprod_{n \in \overline{\mathbb{C}}_{+}, \alpha \in \mathbb{C} / \mathbb{Z}} W_{[n]}^{[\alpha]}$ be a $U_{g}(V)$-module equipped with gradings by $\overline{\mathbb{C}}_{+}$and $\mathbb{C} / \mathbb{Z}$ and an action of $g$ on $W$ satisfying the following conditions:

1. The $U_{g}(V)$-module structure is compatible with the action of $g$ on $U_{g}(V)$ and $W$ in the sense that for $u \in U_{g}(V)$ and $w \in W, g(u w)=(g u)(g w)$.

2. For $u \in V^{[a+\mathbb{Z}]}(a \in P(V))$ and $m \in \mathbb{Z}$ and $w \in W, u(a+m) w=0$ when $m$ is sufficiently large.

3. For $u \in V_{(m)}$ and $w \in W_{p}=\coprod_{\alpha \in \mathbb{C} / \mathbb{Z}} W_{p}^{[\alpha]}$ where $m \in \mathbb{Z}$ and $p \in \overline{\mathbb{C}}_{+}$, $u(n) w$ is 0 when $m-n-1+p \notin \overline{\mathbb{C}}_{+}$, is in $W_{m-n-1+p}$ when $m-n-1+p \in \overline{\mathbb{C}}_{+}$and for $r \in \mathbb{R}, \coprod_{r \in \mathbb{R}} W_{\sqrt{-1} r}$ is equal to the subspace of $W$ consisting of $w \in W$ such that when $u(n) w=0$ for $u \in V_{(m)}, m \in \mathbb{Z}, n \in \mathbb{C}, m-n-1 \notin \overline{\mathbb{C}}_{+}$. For $\alpha \in \mathbb{C} / \mathbb{Z}$, $w \in W^{[\alpha]}=\coprod_{n \in \overline{\mathbb{C}}_{+}} W_{n}^{[\alpha]}$, there exists $\Lambda \in \mathbb{Z}_{+}$such that $\left(g-e^{2 \pi \sqrt{-1} \alpha}\right)^{\Lambda} w=0$, where $L_{g}(0)=\omega(1) \in U_{g}(V)$.

Then $W$ is a $\overline{\mathbb{C}}_{+}$-graded weak g-twisted $V$-module. In particular, the category of $\overline{\mathbb{C}}_{+}$-graded weak g-twisted $V$-modules and the category of $U_{g}(V)$-modules satisfying three conditions above are isomorphic (not just equivalent since the underlying vector spaces are the same). Under this isomorphism, lower bounded generalized $g$-twisted $V$-modules correspond to $U_{g}(V)$ modules of the form $W=\coprod_{n \in \mathbb{C}, \alpha \in \mathbb{C} / \mathbb{Z}} W_{[n]}^{[\alpha]}$ such that $W_{[n]}=\coprod_{\alpha \in \mathbb{C} / Z} W_{[n]}^{[\alpha]}$ for $n \in \mathbb{C}$ are generalized eigenspaces of $L_{g}(0)$, satisfying the three conditions above and the lower bounded condition that for each $n \in \mathbb{C}$ and each $\alpha \in \mathbb{C} / \mathbb{Z}, W_{[n+l]}^{[\alpha]}=0$ for sufficiently negative real number $l$. Under this isomorphism, grading-restricted generalized g-twisted $V$-modules (or simply $g$-twisted $V$-modules as we have called them) correspond to $U_{g}(V)$-modules of the form above, satisfying the three conditions above, the lower bounded condition and the condition that for each $n \in \mathbb{C}, \alpha \in \mathbb{C} / \mathbb{Z}, \operatorname{dim} W_{[n]}^{[\alpha]}<\infty$.

Proof. The first conclusion is clear from the construction of $U_{g}(V)$. 
For its converse, we define $Y_{0}^{g}(u, x) w=\sum_{n \in \alpha+\mathbb{Z}} u(n) w x^{-n-1}$ for $u \in V$ and $w \in W$ and $Y^{g}(u, x) w=Y_{0}^{g}\left(x^{\mathcal{N}} u, x\right) w$. For $u \in V^{[a]}, m \in a+\mathbb{Z}$ and $w \in W^{[b+\mathbb{Z}]}$, we have $u(m) w \in$ $W^{[a+b+\mathbb{Z}]}$ Thus $g Y_{0}^{g}(u, x) w=Y_{0}^{g}(g u, x) g w$ and $g Y^{g}(u, x) w=Y^{g}(g u, x) g w$. Since $u(a+$ $m) w=0$ when $m \in \mathbb{Z}$ is sufficiently large, $Y_{0}^{g}(u, x) w$ is lower truncated. Since $W$ is a $U_{g}(V)$-module, the Jacobi identity (2.29) and the $L(-1)$-derivative property (2.18) hold. By Theorem 2.10, the duality property for $Y^{g}$ holds. It is also clear that all the other axioms for a $g$-twisted generalized $V$-module hold.

The other conclusions are all clear.

It is clear that the subspace $U_{g}(V)_{\mathbb{I}}=\coprod_{n \in \mathbb{I}} U_{g}(V)_{n}$ spanned by elements of $U_{g}(V)$ whose weights are imaginary numbers is a subalgebra of $U_{g}(V)$. Let $N\left(U_{g}(V)_{\mathbb{I}}\right)$ be the two-sided ideal in $U_{g}(V)_{\mathbb{I}}$ generated by

$$
\coprod_{n \in \mathbb{I}, a \in \tilde{P}(V), i \in \mathbb{N}, \Re(a+i)>0} U_{g}(V)_{n+a+i} U_{g}(V)_{-a-i} .
$$

Definition 3.6 The g-twisted zero-mode algebra associated to $V$ is the quotient algebra

$$
Z_{g}(V)=U_{g}(V)_{\mathbb{I}} / N\left(U_{g}(V)_{\mathbb{I}}\right) .
$$

For weight homogeneous $v \in V^{[a+\mathbb{Z}]}, a \in P(V) \cap \mathbb{I}$, we write $o_{g}(v)$ for $v($ wt $v+a-1) \in$ $U_{g}(V)_{\mathbb{I}}$, denote by $\left[o_{g}(v)\right]$ the image of $o_{g}(v)$ in the quotient $Z_{g}(V)$, and extend $\left[o_{g}(v)\right]$ to all $v \in V$ by linearity.

From the definition of $Z_{g}(V)$ and Proposition 3.4, we have the following useful result:

Proposition 3.7 The g-twisted zero-mode algebra $Z_{g}(V)$ is spanned by elements of the form

$$
\left[o_{g}\left(u_{1}\right)\right] \cdots\left[o_{g}\left(u_{k}\right)\right]
$$

for $u_{1}, \ldots, u_{k} \in V$ and $k \in \mathbb{N}$.

Proof. By Proposition 3.4, elements of $U_{g}(V)_{n}$ for $n \in \mathbb{I}$ are of the form $u_{1}+u_{2}$ where $u_{1} \in \pi_{U_{g}(V)} T(L(V, g))_{n}$, and $u_{2}$ is in the topological completion of

$$
\coprod_{a \in \tilde{P}(V), i \in \mathbb{Z}_{+}}\left(\pi_{U_{g}(V)} T(L(V, g))_{n+a+i}\right)\left(\pi_{U_{g}(V)} T(L(V, g))_{-a-i}\right) .
$$

Since $\left(\pi_{U_{g}(V)} T(L(V, g))_{n+a+i}\right)\left(\pi_{U_{g}(V)} T(L(V, g))_{-a-i}\right) \subset U_{g}(V)_{n+a+i} U_{g}(V)_{-a-i}$, we see that $u_{2}$ is in the topological completion of $\coprod_{a \in \tilde{P}(V), i \in \mathbb{Z}_{+}} U_{g}(V)_{n+a+i} U_{g}(V)_{-a-i}$. Since $\Re(a+i)>0$ for $a \in \tilde{P}(V)$ and $i \in \mathbb{Z}_{+}$, we have $\coprod_{a \in \tilde{P}(V), i \in \mathbb{Z}_{+}} U_{g}(V)_{n+a+i} U_{g}(V)_{-a-i} \subset N\left(U_{g}(V)_{\mathbb{I}}\right)$. Thus $u_{2} \in$ $N\left(U_{g}(V)_{\mathbb{I}}\right)$ and elements of $Z_{g}(V)$ are of the form $u_{1}+N\left(U_{g}(V)_{\mathbb{I}}\right)$ for $u_{1} \in \pi_{U_{g}(V)} T(L(V, g))_{n}$, $n \in \mathbb{I}$. But $T(L(V, g))_{n}$ is spanned by elements of the form $u_{1}\left(m_{1}\right) \cdots u_{k}\left(m_{k}\right)$ for $k \in \mathbb{N}$, homogeneous $u_{1} \in V^{\left[a_{1}+\mathbb{Z}\right]}, \ldots, u_{k} \in V^{\left[a_{k}+\mathbb{Z}\right]}, a_{1}, \ldots, a_{k} \in P(V), m_{1} \in a_{1}+\mathbb{Z}, \ldots, m_{k} \in a_{k}+\mathbb{Z}$ such that $\sum_{i=1}^{k}\left(\right.$ wt $\left.u_{i}-m_{i}-1\right)=n$. For simplicity, we shall also use the same notation to 
denote the images of such elements in $U_{g}(V)_{n}$ under the map $\pi_{U_{g}(V)}$. Since the commutator formula holds for elements in $U_{g}(V)$, these elements in $U_{g}(V)_{n}$ are linear combinations of elements of the same form but satisfying the additional condition $\Re\left(\right.$ wt $\left.u_{i}-m_{1}-1\right) \geq \cdots \geq$ $\Re$ (wt $u_{i}-m_{k}-1$ ). If $\Re\left(\right.$ wt $\left.u_{i}-m_{i}-1\right)<0$ for some $i$, then $\Re$ (wt $\left.u_{k}-m_{k}-1\right)<0$ and $u_{1}\left(m_{1}\right) \cdots u_{k}\left(m_{k}\right)$ is in $\left.\left.U_{g}(V)_{n+a_{k}+(-w t} u_{k}+m_{k}-a_{k}+1\right) U_{g}(V)_{-a_{k}-(-w t} u_{k}+m_{k}-a_{k}+1\right)$ satisfying $\Re\left(a_{k}+\left(\right.\right.$ wt $\left.\left.u_{k}+m_{k}-a_{k}+1\right)\right)=\Re\left(-\right.$ wt $\left.u_{k}+m_{k}+1\right)>0$. Hence $u_{1}\left(m_{1}\right) \cdots u_{k}\left(m_{k}\right)$ is in $N\left(U_{g}(V)_{\mathbb{I}}\right)$. Thus if $u_{1}\left(m_{1}\right) \cdots u_{k}\left(m_{k}\right)$ is not in $N\left(U_{g}(V)_{\mathbb{I}}\right), \Re\left(\right.$ wt $\left.u_{i}-m_{i}-1\right) \geq 0$ for $i=1, \ldots, k$. Since $\sum_{i=1}^{k}\left(\right.$ wt $\left.u_{i}-m_{i}-1\right)=n \in \mathbb{I}$, we must have $\Re\left(\right.$ wt $\left.u_{i}-m_{i}-1\right)=0$ or $\Re\left(m_{i}\right)=$ wt $u_{i}-1$ for $i=1, \ldots, k$. Since $m_{i} \in a_{i}+\mathbb{Z}$, we see that $\Re\left(a_{i}\right)=0$ and hence $a_{i} \in \mathbb{I}$. Moreover, $m_{i}=$ wt $u_{i}+a_{i}-1$. Thus

$$
u_{1}\left(m_{1}\right) \cdots u_{k}\left(m_{k}\right)+N\left(U_{g}(V)_{\mathbb{I}}\right)=\left[o_{g}\left(u_{1}\right)\right] \cdots\left[o_{g}\left(u_{k}\right)\right]
$$

We shall discuss modules for $U_{g}(V)$ and for $Z_{g}(V)$ and their connections with suitable $g$-twisted $V$-modules in Section 5. In Section 6, Proposition 3.7 is needed in the proof that $Z_{g}(V)$ and $A_{g}(V)$ ( (to be constructed in the next section) are isomorphic. Then as a consequence of the isomorphism, we obtain in turn an improvement of Proposition 3.7.

\section{A generalization of Zhu's algebra to the $g$-twisted case for $g$ of infinite order}

In [DLM1, Dong, Li and Mason introduced a generalization of Zhu's algebra for suitable $g$-twisted $V$-modules when $g$ is of finite order. But their generalization does not generalize straightforwardly to the case of a general automorphism $g$, especially in the case that $g$ does not act on $V$ semisimply. In this section, we give such a generalization. When $g$ does not act on $V$ semisimply, the definition of this associative algebra involves the operator $\mathcal{N}$ obtained from the multiplicative Jordan-Chevalley decomposition of $g$. Moreover, this associative algebra is not a quotient of the fixed point subalgebra; instead, it is a quotient of the generalized eigenspace of eigenvalue 1 of $g$. This means that when $g$ does not act on $V$ semisimply (in this case the order of $g$ must be infinite), this generalization of Zhu's algebra might have some interesting new features.

In [H2], the first author found a natural definition of Zhu's algebra in connection with the modular invariance. Besides its geometric meaning in connection with the modular invariance, the advantage of this definition is that one can separate vertex operators from other formal series or analytic functions in the proofs of the properties of the algebra. This advantage makes it particularly simple for our generalization such that all the proofs are identical to those in [H2] except for some properties of the operator $\mathcal{N}$. Because of this reason, we give our generalization by generalizing the definition in [H2]. Just as in [H2], the difference between the definition given in this section and a direct generalization of

the definitions of Zhu and Dong-Li-Mason is given by an isomorphism obtained from the 
conformal transformation $\frac{1}{2 \pi i} \log (1+2 \pi i z)$, the geometry underlying vertex operators and the Virasoro operators.

For weight homogeneous $u \in V^{[a+\mathbb{Z}]}, a \in P(V)$, let $l(u) \in a+\mathbb{Z}$ such that $\Re($ wt $u-n-1)<$ 0 for $n \in a+\mathbb{Z}$ and $n-a \geq l(u)-a$. Then $l(u)=$ wt $u+a$ when $a \in P(V) \cap \mathbb{I}$ and $l(u)=$ wt $u+a-1$ when $a \in P(V) \cap((0,1)+\mathbb{I})$. We define a linear operator $\mathbf{L}$ on $V$ by $\mathbf{L} u=l(u) u$ for weight homogeneous $u \in V^{[a+\mathbb{Z}]}$ and linearity. We define a product $\bullet_{g}$ in $V$ as follows: For $u, v \in V$,

$$
\begin{aligned}
u \bullet g & =\operatorname{Res}_{y} y^{-1} Y\left((1+y)^{\mathbf{L}-L(0)+\mathcal{N}} u, \frac{1}{2 \pi \sqrt{-1}} \log (1+y)\right) v \\
& =\operatorname{Res}_{x} \frac{2 \pi \sqrt{-1} e^{2 \pi \sqrt{-1} x}}{e^{2 \pi \sqrt{-1}}-1} Y\left(e^{2 \pi \sqrt{-1} x(\mathbf{L}-L(0)+\mathcal{N})} u, x\right) v .
\end{aligned}
$$

Recall the operator $\mathbf{A}_{V}$ defined in Section 2. Let $\tilde{O}_{g}(V)$ be the subspace of $V$ spanned elements of the form

$$
\begin{aligned}
& \operatorname{Res}_{y} y^{-n} Y\left(y^{L(0)-\mathbf{L}+\mathbf{A}_{V}}(1+y)^{\mathbf{L}-L(0)+\mathcal{N}} u, \frac{1}{2 \pi \sqrt{-1}} \log (1+y)\right) v \\
& =\operatorname{Res}_{x} \frac{2 \pi \sqrt{-1} e^{2 \pi \sqrt{-1} x}}{\left(e^{2 \pi \sqrt{-1} x}-1\right)^{n}} Y\left(\left(\frac{1}{e^{2 \pi \sqrt{-1} x}-1}\right)^{L(0)-\mathbf{L}+\mathbf{A}_{V}} e^{2 \pi \sqrt{-1} x(\mathbf{L}-L(0)+\mathcal{N})} u, x\right) v
\end{aligned}
$$

for $n>1, u, v \in V$. Note that by definition, when $u \in V^{[\alpha]}, a \notin \mathbb{I}, u \bullet_{g} v \in \tilde{O}_{g}(V)$. Also note that

$$
\operatorname{Res}_{y} y^{-n} Y\left((1+y)^{\mathbf{L}-L(0)+\mathcal{N}} u, \frac{1}{2 \pi \sqrt{-1}} \log (1+y)\right) v \in \tilde{O}_{g}(V)
$$

for $n>1$ since for homogeneous $u,\left(L(0)-\mathbf{L}+\mathbf{A}_{V}\right) u$ is either 0 or $u$.

Let $\tilde{A}_{g}(V)=V / \tilde{O}_{g}(V)$.

Theorem 4.1 The product $\bullet_{g}$ in $V$ induces a product (denoted still by $\bullet_{g}$ ) in $\tilde{A}_{g}(V)$ such that $\tilde{A}_{g}(V)$ together with this product and the equivalence class of the vacuum $\mathbf{1} \in V$ is an associative algebra with identity. Moreover, for $u \in V^{[a+\mathbb{Z}]}, a \in P(V) \cap \mathbb{I}, v \in V$, $((L(-1)+2 \pi \sqrt{-1}(a+\mathcal{N})) u) \bullet g v \in \tilde{O}_{g}(V)$ and for $u \in V^{[a+\mathbb{Z}]}, v \in V^{[b+\mathbb{Z}]}, a, b \in P(V) \cap \mathbb{I}$,

$$
\begin{aligned}
u \bullet_{g} v-v \bullet_{g} u & \equiv 2 \pi \sqrt{-1} \operatorname{Res}_{x} Y\left(e^{2 \pi \sqrt{-1} x(a+\mathcal{N})} u, x\right) v \quad \bmod \tilde{O}_{g}(V) \\
& =2 \pi \sqrt{-1} \sum_{i \in \mathbb{N}} \frac{2 \pi \sqrt{-1}}{i !}\left((\mathcal{N}+a)^{i} u\right)_{i} v .
\end{aligned}
$$

In particular, $\omega \bullet_{g} v-v \bullet_{g} \omega \equiv(2 \pi \sqrt{-1})^{2} \mathcal{N} v \bmod \tilde{O}_{g}(V)$ for $v \in V^{[b+\mathbb{Z}]}, b \in P(V) \cap \mathbb{I}$, and $\omega+\tilde{O}_{g}(V)$ brackets with $v+\tilde{O}_{g}(V)$ sufficiently many times in $\tilde{A}_{g}(V)$ is 0 for $v \in V$.

Proof. The proof of the first part is the same as the proof of the corresponding part in Proposition 6.1 in [H2] except that we need to use (2.14) to take care of the additional 
operator $e^{2 \pi \sqrt{-1} x \mathcal{N}}$ appearing in the definition and to take care of those factors involving $a \in P(V)$ just as in [DLM1]. To convince the reader that the same proof together with (2.14) and with the adjustments involving $a \in P(V)$ indeed works, we give as examples the proof that

$$
\begin{gathered}
\left(\operatorname{Res}_{x} \frac{2 \pi \sqrt{-1} e^{2 \pi \sqrt{-1} x}}{\left(e^{2 \pi \sqrt{-1} x}-1\right)^{n}} Y\left(\left(\frac{1}{e^{2 \pi \sqrt{-1} x}-1}\right)^{L(0)-\mathbf{L}+\mathbf{A}_{V}} e^{2 \pi \sqrt{-1} x(\mathbf{L}-L(0)+\mathcal{N})} u_{1}, x\right) u_{2}\right) \bullet_{g} u_{3} \\
=\left(\operatorname{Res}_{x} \frac{2 \pi \sqrt{-1} e^{2 \pi \sqrt{-1} a x}}{\left(e^{2 \pi \sqrt{-1} x}-1\right)^{n}} Y\left(e^{2 \pi \sqrt{-1} x \mathcal{N}} u_{1}, x\right) u_{2}\right) \bullet_{g} u_{3}
\end{gathered}
$$

is in $\tilde{O}_{g}(V)$ when $u_{1} \in V^{[a+\mathbb{Z}]}(a \neq 0), u_{2}, u_{3} \in V$ and $n>1$.

In the case that $u_{2} \in V^{[b+\mathbb{Z}]}$ and $b \neq 1-a$,

$$
\operatorname{Res}_{x} \frac{2 \pi \sqrt{-1} e^{2 \pi \sqrt{-1} a x}}{\left(e^{2 \pi \sqrt{-1} x}-1\right)^{n}} Y\left(e^{2 \pi \sqrt{-1} x \mathcal{N}} u_{1}, x\right) u_{2} \in V^{[a+b+\mathbb{Z}]} \neq V^{[0]} .
$$

Thus by definition, (4.3) is in $\tilde{O}_{g}(V)$. The only case we need to consider is $u_{2} \in V^{[1-a+\mathbb{Z}]}$.

Using the definition of $\bullet_{g}$, (2.14), the Jacobi identity for the vertex operator for $V$ and the property of the formal delta function, we modify the first step in (6.8) in [H2] to obtain that (4.3) is equal to

$$
\begin{aligned}
& \operatorname{Res}_{x_{0}} \operatorname{Res}_{x_{2}} \frac{2 \pi \sqrt{-1} e^{2 \pi \sqrt{-1} a x_{0}}}{\left(e^{2 \pi \sqrt{-1} x_{0}}-1\right)^{n}} \frac{2 \pi \sqrt{-1} e^{2 \pi \sqrt{-1} x_{2}}}{e^{2 \pi \sqrt{-1} x_{2}}-1} Y\left(e^{2 \pi \sqrt{-1} x_{2} \mathcal{N}} Y\left(e^{2 \pi \sqrt{-1} x_{0} \mathcal{N}} u_{1}, x_{0}\right) u_{2}, x_{2}\right) u_{3} \\
& =\operatorname{Res}_{x_{0}} \operatorname{Res}_{x_{2}} \frac{2 \pi \sqrt{-1} e^{2 \pi \sqrt{-1} a x_{0}}}{\left(e^{2 \pi \sqrt{-1} x_{0}}-1\right)^{n}} \frac{2 \pi \sqrt{-1} e^{2 \pi \sqrt{-1} x_{2}}}{e^{2 \pi \sqrt{-1} x_{2}}-1} . \\
& \cdot Y\left(Y\left(e^{2 \pi \sqrt{-1}\left(x_{2}+x_{0}\right) \mathcal{N}} u_{1}, x_{0}\right) e^{2 \pi \sqrt{-1} x_{2} \mathcal{N}} u_{2}, x_{2}\right) u_{3} \\
& =\operatorname{Res}_{x_{1}} \operatorname{Res}_{x_{2}} \operatorname{Res}_{x_{0}} \frac{2 \pi \sqrt{-1} e^{2 \pi \sqrt{-1} a x_{0}}}{\left(e^{2 \pi \sqrt{-1} x_{0}}-1\right)^{n}} \frac{2 \pi \sqrt{-1} e^{2 \pi \sqrt{-1} x_{2}}}{e^{2 \pi \sqrt{-1} x_{2}}-1} . \\
& \cdot x_{0}^{-1} \delta\left(\frac{x_{1}-x_{2}}{x_{0}}\right) Y\left(e^{2 \pi \sqrt{-1}\left(x_{2}+x_{0}\right) \mathcal{N}} u_{1}, x_{1}\right) Y\left(e^{2 \pi \sqrt{-1} x_{2} \mathcal{N}} u_{2}, x_{2}\right) u_{3} \\
& -\operatorname{Res}_{x_{1}} \operatorname{Res}_{x_{2}} \operatorname{Res}_{x_{0}} \frac{2 \pi \sqrt{-1} e^{2 \pi \sqrt{-1} a x_{0}}}{\left(e^{2 \pi \sqrt{-1} x_{0}}-1\right)^{n}} \frac{2 \pi \sqrt{-1} e^{2 \pi \sqrt{-1} x_{2}}}{e^{2 \pi \sqrt{-1} x_{2}}-1} . \\
& \cdot x_{0}^{-1} \delta\left(\frac{x_{2}-x_{1}}{-x_{0}}\right) Y\left(e^{2 \pi \sqrt{-1} x_{2} \mathcal{N}} u_{2}, x_{2}\right) Y\left(e^{2 \pi \sqrt{-1}\left(x_{2}+x_{0}\right) \mathcal{N}} u_{1}, x_{1}\right) u_{3} \\
& =\operatorname{Res}_{x_{1}} \operatorname{Res}_{x_{2}} \operatorname{Res}_{x_{0}} \frac{2 \pi \sqrt{-1} e^{2 \pi \sqrt{-1} a x_{0}}}{\left(e^{2 \pi \sqrt{-1} x_{0}}-1\right)^{n}} \frac{2 \pi \sqrt{-1} e^{2 \pi \sqrt{-1} x_{2}}}{e^{2 \pi \sqrt{-1} x_{2}}-1} . \\
& \cdot x_{0}^{-1} \delta\left(\frac{x_{1}-x_{2}}{x_{0}}\right) Y\left(e^{2 \pi \sqrt{-1} x_{1} \mathcal{N}} u_{1}, x_{1}\right) Y\left(e^{2 \pi \sqrt{-1} x_{2} \mathcal{N}} u_{2}, x_{2}\right) u_{3}
\end{aligned}
$$




$$
\begin{aligned}
-\operatorname{Res}_{x_{1}} \operatorname{Res}_{x_{2}} \operatorname{Res}_{x_{0}} \frac{2 \pi \sqrt{-1} e^{2 \pi \sqrt{-1} a x_{0}}}{\left(e^{2 \pi \sqrt{-1} x_{0}}-1\right)^{n}} \frac{2 \pi \sqrt{-1} e^{2 \pi \sqrt{-1} x_{2}}}{e^{2 \pi \sqrt{-1} x_{2}}-1} . \\
\cdot x_{0}^{-1} \delta\left(\frac{x_{2}-x_{1}}{-x_{0}}\right) Y\left(e^{2 \pi \sqrt{-1} x_{2} \mathcal{N}} u_{2}, x_{2}\right) Y\left(e^{2 \pi \sqrt{-1} x_{1} \mathcal{N}} u_{1}, x_{1}\right) u_{3} .
\end{aligned}
$$

Then using the same remaining steps in (6.8) in [H2], we see that the right-hand side of (4.4) is equal to

$$
\begin{aligned}
& \sum_{k \in \mathbb{N}}\left(\begin{array}{c}
-n \\
k
\end{array}\right) \operatorname{Res}_{x_{1}} \operatorname{Res}_{x_{2}} 2 \pi \sqrt{-1} e^{2 \pi \sqrt{-1} a\left(x_{1}-x_{2}\right)}\left(e^{2 \pi \sqrt{-1} x_{1}}-1\right)^{-n-k} e^{-2 \pi \sqrt{-1}(-n-k) x_{2}} . \\
& \cdot\left(e^{-2 \pi \sqrt{-1} x_{2}}-1\right)^{k} \frac{2 \pi \sqrt{-1} e^{2 \pi \sqrt{-1} x_{2}}}{e^{2 \pi \sqrt{-1} x_{2}}-1} Y\left(e^{2 \pi \sqrt{-1} x_{2} \mathcal{N}} u_{1}, x_{1}\right) Y\left(e^{2 \pi \sqrt{-1} x_{2} \mathcal{N}} u_{2}, x_{2}\right) u_{3} \\
& -\sum_{k \in \mathbb{N}}\left(\begin{array}{c}
-n \\
k
\end{array}\right) \operatorname{Res}_{x_{1}} \operatorname{Res}_{x_{2}} 2 \pi \sqrt{-1} e^{-2 \pi \sqrt{-1} a\left(x_{2}-x_{1}\right)}\left(e^{-2 \pi \sqrt{-1} x_{2}}-1\right)^{-n-k} e^{2 \pi \sqrt{-1}(-n-k) x_{1}} . \\
& \cdot\left(e^{2 \pi \sqrt{-1} x_{1}}-1\right)^{k} \frac{2 \pi \sqrt{-1} e^{2 \pi \sqrt{-1} x_{2}}}{e^{2 \pi \sqrt{-1} x_{2}}-1} Y\left(e^{2 \pi \sqrt{-1} x_{2} \mathcal{N}} u_{2}, x_{2}\right) Y\left(e^{2 \pi \sqrt{-1} x_{2} \mathcal{N}} u_{1}, x_{1}\right) u_{3} \\
& =\sum_{k \in \mathbb{N}}\left(\begin{array}{c}
-n \\
k
\end{array}\right) \operatorname{Res}_{x_{2}} e^{-2 \pi \sqrt{-1} a x_{2}} e^{-2 \pi \sqrt{-1}(-n-k) x_{2}}\left(e^{-2 \pi \sqrt{-1} x_{2}}-1\right)^{k} \frac{2 \pi \sqrt{-1} e^{2 \pi \sqrt{-1} x_{2}}}{e^{2 \pi \sqrt{-1} x_{2}}-1} . \\
& \cdot \operatorname{Res}_{x_{1}} \frac{2 \pi \sqrt{-1} e^{2 \pi \sqrt{-1} a x_{1}}}{\left(e^{2 \pi \sqrt{-1} x_{1}}-1\right)^{n+k}} Y\left(e^{2 \pi \sqrt{-1} x_{2} \mathcal{N}} u_{1}, x_{1}\right) Y\left(e^{2 \pi \sqrt{-1} x_{2} \mathcal{N}} u_{2}, x_{2}\right) u_{3} \\
& -\sum_{k \in \mathbb{N}}\left(\begin{array}{c}
-n \\
k
\end{array}\right) \operatorname{Res}_{x_{1}} e^{2 \pi \sqrt{-1} a x_{1}}(-1)^{-n-k} e^{2 \pi \sqrt{-1}(-n-k) x_{1}}\left(e^{2 \pi \sqrt{-1} x_{1}}-1\right)^{k} . \\
& \cdot \operatorname{Res}_{x_{2}} \frac{2 \pi \sqrt{-1} e^{2 \pi \sqrt{-1}(1-a) x_{2}}}{\left(e^{2 \pi \sqrt{-1}} x_{2}-1\right)^{1+n+k}} e^{2 \pi \sqrt{-1}(n+k) x_{2}} Y\left(e^{2 \pi \sqrt{-1} x_{2} \mathcal{N}} u_{2}, x_{2}\right) Y\left(e^{2 \pi \sqrt{-1} x_{2} \mathcal{N}} u_{1}, x_{1}\right) u_{3} .
\end{aligned}
$$

The first term in the right-hand side of (4.5) is in $\tilde{O}_{g}(V)$. Since $u_{2} \in V^{[1-a+\mathbb{Z}]} \neq V^{[0]}$ and $e^{2 \pi \sqrt{-1}(n+k) x_{2}}$ can be written as a polynomial in $e^{2 \pi \sqrt{-1} x_{2}}-1$ with degree less than or equal to $n+k$, the second term in the right-hand side of (4.5) is also in $\tilde{O}_{g}(V)$.

Next we prove $((L(-1)+2 \pi \sqrt{-1}(a+\mathcal{N})) u) \bullet_{g} v \in \tilde{O}_{g}(V)$ for $u \in V^{[a+\mathbb{Z}]}, a \in P(V) \cap \mathbb{I}$ and $v \in V$. Using the properties of the vertex operator map $Y$ and (2.16), we have

$$
\begin{aligned}
& (L(-1) u) \bullet_{g} v \\
& =\operatorname{Res}_{x} \frac{2 \pi \sqrt{-1} e^{2 \pi \sqrt{-1} x}}{e^{2 \pi \sqrt{-1} x}-1} Y\left(e^{2 \pi \sqrt{-1} x(a+\mathcal{N})} L(-1) u, x\right) v \\
& =\operatorname{Res}_{x} \frac{2 \pi \sqrt{-1} e^{2 \pi \sqrt{-1} x}}{e^{2 \pi \sqrt{-1} x}-1} \frac{d}{d x} Y\left(e^{2 \pi \sqrt{-1} x(a+\mathcal{N})} u, x\right) v \\
& \quad-\operatorname{Res}_{x} \frac{2 \pi \sqrt{-1} e^{2 \pi \sqrt{-1} x}}{e^{2 \pi \sqrt{-1}}-1} Y\left(e^{2 \pi \sqrt{-1} x(a+\mathcal{N})} 2 \pi \sqrt{-1}(a+\mathcal{N}) u, x\right) v
\end{aligned}
$$




$$
\begin{aligned}
& =-\operatorname{Res}_{x}\left(\frac{d}{d x} \frac{2 \pi \sqrt{-1} e^{2 \pi \sqrt{-1} x}}{e^{2 \pi \sqrt{-1} x}-1}\right) Y\left(e^{2 \pi \sqrt{-1} x(a+\mathcal{N})} u, x\right) v-2 \pi \sqrt{-1}((a+\mathcal{N}) u) \bullet v \\
& =\operatorname{Res}_{x} \frac{(2 \pi \sqrt{-1})^{2} e^{2 \pi \sqrt{-1} x}}{\left(e^{2 \pi \sqrt{-1} x}-1\right)^{2}} Y\left(e^{2 \pi \sqrt{-1} x \mathcal{N}} v, x\right) \mathbf{1}-2 \pi \sqrt{-1}((a+\mathcal{N}) u) \bullet v .
\end{aligned}
$$

Since the first term in (4.6) is in $\tilde{O}_{g}(V)$, we obtain $((L(-1)+2 \pi \sqrt{-1}(a+\mathcal{N})) u) \bullet_{g} v \in \tilde{O}_{g}(V)$. Now we prove (4.2). For $u \in V^{[a+\mathbb{Z}]}, v \in V^{[b+\mathbb{Z}]}, a, b \in P(V) \cap \mathbb{I}$,

$$
\begin{array}{rl}
u \bullet g & v-2 \pi \sqrt{-1} \operatorname{Res}_{x} Y\left(e^{2 \pi \sqrt{-1} x(a+\mathcal{N})} u, x\right) v \\
& =\operatorname{Res}_{x} \frac{2 \pi \sqrt{-1} e^{2 \pi \sqrt{-1} x}}{e^{2 \pi \sqrt{-1} x}-1} Y\left(e^{2 \pi \sqrt{-1} x(a+\mathcal{N})} u, x\right) v-2 \pi \sqrt{-1} \operatorname{Res}_{x} Y\left(e^{2 \pi \sqrt{-1} x(a+\mathcal{N})} u, x\right) v \\
& =-\operatorname{Res}_{x} \frac{2 \pi \sqrt{-1} e^{-2 \pi \sqrt{-1} x}}{e^{-2 \pi \sqrt{-1} x}-1} Y\left(e^{2 \pi \sqrt{-1} x(a+\mathcal{N})} u, x\right) v \\
& =-\operatorname{Res}_{x} \frac{2 \pi \sqrt{-1} e^{-2 \pi \sqrt{-1}} x}{e^{-2 \pi \sqrt{-1} x}-1} e^{2 \pi \sqrt{-1} x(a+b+\mathcal{N})} Y(u, x) e^{-2 \pi \sqrt{-1} x(b+\mathcal{N})} v \\
& \equiv-\operatorname{Res}_{x} \frac{2 \pi \sqrt{-1} e^{-2 \pi \sqrt{-1} x}}{e^{-2 \pi \sqrt{-1} x}-1} e^{-x L(-1)} Y(u, x) e^{-2 \pi \sqrt{-1} x(b+\mathcal{N})} v \quad \bmod \tilde{O}_{g}(V) \\
& =-\operatorname{Res}_{x} \frac{2 \pi \sqrt{-1} e^{-2 \pi \sqrt{-1} x}}{e^{-2 \pi \sqrt{-1} x}-1} Y\left(e^{-2 \pi \sqrt{-1} x(b+\mathcal{N})} v,-x\right) u \\
& =\operatorname{Res}_{y} \frac{2 \pi \sqrt{-1} e^{2 \pi \sqrt{-1} y}}{e^{2 \pi \sqrt{-1} y}-1} Y\left(e^{2 \pi \sqrt{-1} y(b+\mathcal{N})} v, y\right) u \\
& =v \bullet \bullet_{g} u
\end{array}
$$

where we have used (2.14), the fact that the coefficients of $Y(u, x) e^{-2 \pi \sqrt{-1} x(b+\mathcal{N})} v$ is in $V^{[a+b+\mathbb{Z}]},(4.6)$ with $a$ replaced by $a+b$ and the skew-symmetry of $Y$.

Take $u=\omega$ and note that $\mathcal{N} \omega=0, a=0$ and $\omega_{0}=L(-1)$. Then we obtain $\omega \bullet \bullet_{g} v-v \bullet g=$ $L(-1) v \equiv-2 \pi \sqrt{-1} \mathcal{N} v \bmod \tilde{O}_{g}(V)$. Since $\mathcal{N}$ is nilpotent, we see that the last conclusion is true.

We now derive a generalization of the algebra $A_{g}(V)$ introduced in [DLM1]. Recall the invertible operator

$$
\mathcal{U}(1)=(2 \pi \sqrt{-1})^{L(0)} e^{-L_{+}(A)}
$$

on $V$ introduced in [H2], where $L_{+}(A)=\sum_{n \in \mathbb{Z}_{+}} A_{j} L(j)$ and the coeffieients $A_{j}$ for $j \in \mathbb{Z}_{+}$ are uniquely determined by

$$
\frac{1}{2 \pi \sqrt{-1}}\left(e^{2 \pi \sqrt{-1} y}-1\right)=\left(\exp \left(-\sum_{j \in \mathbb{Z}_{+}} A_{j} y^{j+1} \frac{\partial}{\partial y}\right)\right) y
$$


Note that $\mathcal{N}$ commutes with all the Virasoro operators (see (2.16) ). Thus (1.5) in [H2] gives

$$
\begin{gathered}
\mathcal{U}(1) \operatorname{Res}_{y} y^{-n} Y\left((1+y)^{\mathcal{N}} u, \frac{1}{2 \pi \sqrt{-1}} \log (1+y)\right) v \\
=\operatorname{Res}_{y} y^{-n} Y\left((1+y)^{L(0)+\mathcal{N}} \mathcal{U}(1) u, y\right) \mathcal{U}(1) v
\end{gathered}
$$

We define a product $*$ on $V$ by

$$
u *_{g} v=\operatorname{Res}_{x} x^{-1} Y\left((1+x)^{\mathbf{L}+\mathcal{N}} u, x\right) v
$$

for $u, v \in V$. Let $O_{g}(V)$ be the subspace of $V$ spanned by elements of the form

$$
\operatorname{Res}_{x} x^{-n} Y\left(x^{L(0)-\mathbf{L}+\mathbf{A}_{V}}(1+x)^{\mathbf{L}+\mathcal{N}} u, x\right) v
$$

for $n>1, u, v \in V$. By (4.7), we obtain the following result:

Corollary 4.2 Let $A_{g}(V)=V / O_{g}(V)$. Then $A_{g}(V)$ is an associative algebra isomorphic to $\tilde{A}_{g}(V)$. The isomorphism from $\tilde{A}_{g}(V)$ to $A_{g}(V)$ is given by the map induced from $\mathcal{U}(1)$.

Because of Corollary 4.2 , the categories of $A_{g}(V)$-modules and $\tilde{A}_{g}(V)$-modules are equivalent.

Since the map $\mathcal{U}(1)$ corresponds to the coordinate change from the standard coordinate on an annulus centered at 0 in the sphere $\mathbb{C} \cup\{0\}$ to the standard coordinate on the corresponding parallelogram, the algebra $\tilde{A}_{g}(V)$ defined using this coordinate change is most suitable for the study of the modular invariance (see [H2]).

\section{$5 \quad$ Functors between module categories}

In this section, we construct functors between suitable categories of $Z_{g}(V)$-modules, $\tilde{A}_{g}(V)$ modules, $A_{g}(V)$-modules and $g$-twisted $V$-modules.

We are interested only in $Z_{g}(V)$-modules that have compatible actions of $g$ and can be written as direct sums of generalized eigenspaces of the actions of $g$. Recall the notation $\left[o_{g}(v)\right]$ for $v \in V$ in Section 3. We are also interested in such $Z_{g}(V)$-modules with another grading given by the generalized eigenspaces of $\left[o_{g}(\omega)\right] \in Z_{g}(V)$.

Definition 5.1 Let $M$ be a $Z_{g}(V)$-module. If $M=\coprod_{\alpha \in \mathbb{C} / \mathbb{Z}} M^{[\alpha]}$ where $M^{[\alpha]}$ for $\alpha \in \mathbb{C} / \mathbb{Z}$ are the generalized eigenspaces of the action of $g$, we call $M$ a g-graded $Z_{g}(V)$-module. Let $M=\coprod_{n \in \mathbb{C}, \alpha \in \mathbb{C} / \mathbb{Z}} M_{[n]}^{[\alpha]}$ be a $Z_{g}(V)$-module with double gradings by $\mathbb{C}$ and by $\mathbb{C} / \mathbb{Z}$ (or equivalently, by a subset of $[0,1)$ ). If $M_{[n]}=\coprod_{\alpha \in \mathbb{C} / \mathbb{Z}} M_{[n]}^{[\alpha]}$ for $n \in \mathbb{C}$ are generalized eigenspaces of the action of $\left[o_{g}(\omega)\right]$ on $M$ with eigenvalues $n$ (called weights) and the $\mathbb{C} / \mathbb{Z}$ grading is given by the generalized eigenspaces of an action of $g$ compatible with the $Z_{g}(V)$ module structure, then we call $M$ a doubly-graded $Z_{g}(V)$-module or simply graded $Z_{g}(V)$ module. Let $M=\coprod_{n \in \mathbb{C}, \alpha \in \mathbb{C} / \mathbb{Z}} M_{[n]}^{[\alpha]}$ be a graded $Z_{g}(V)$-module, if for each $n \in \mathbb{C}$ and each 
$\alpha \in \mathbb{C} / \mathbb{Z}, M_{[n+l]}^{[\alpha]}=0$ for sufficiently negative real number $l$, we call $M$ a lower bounded $Z_{g}(V)$ module. A lower bounded $Z_{g}(V)$-module $M=\coprod_{n \in \mathbb{C}, \alpha \in \mathbb{C} / \mathbb{Z}} M_{[n]}^{[\alpha]}$ is called grading restricted if $\operatorname{dim} M_{[n]}^{[\alpha]}<\infty$ for $n \in \mathbb{C}, \alpha \in \mathbb{C} / \mathbb{Z}$.

Note that in particular, $Z_{g}(V)$ itself is a $g$-graded $Z_{g}(V)$-module.

Let $W=\coprod_{n \in \overline{\mathbb{C}}_{+}, \alpha \in \mathbb{C} / \mathbb{Z}} W_{n}^{[\alpha]}$ be a $\overline{\mathbb{C}}_{+}$-graded weak $g$-twisted $V$-module. Recall the set $P(W)$ of all $g$-weights of $W$. In particular, $W=\coprod_{n \in \overline{\mathbb{C}}_{+}, a \in P(W)} W_{n}^{[a]}$. It is clear that for $a \in P(V)$ and $b \in P(W)$, either $a+b \in P(W)$ or $a+b-1 \in P(W)$. Let

$$
\Omega^{g}(W)=\{w \in W \mid u(k) w=0 \text { for homogeneous } u \in V, \Re(\text { wt } u-k-1)<0\} .
$$

Then by the $\overline{\mathbb{C}}_{+}$-grading condition in Definition 2.1 and (2.10), $\Omega^{g}(W)=\coprod_{n \in \mathbb{I}} W_{n}=$ $\coprod_{n \in \mathbb{I}, a \in P(W)} W_{n}^{[a+\mathbb{Z}]}$. In the case that $W$ is a lower bounded $g$-twisted $V$-module, the gradings on $W$ also induce gradings on $\Omega^{g}(W)$. In particular, we have

$$
\Omega^{g}(W)=\coprod_{n \in \mathbb{C}}\left(\Omega^{g}(W)\right)_{[n]}=\coprod_{a \in P(W)}\left(\Omega^{g}(W)\right)^{[a+\mathbb{Z}]}=\coprod_{n \in \mathbb{C}, a \in P(W)}\left(\Omega^{g}(W)\right)_{[n]}^{[a+\mathbb{Z}]} .
$$

By Theorem 3.5, $W$ is a $U_{g}(V)$-module and hence also a $U_{g}(V)_{\mathbb{I}}$-module. In particular,

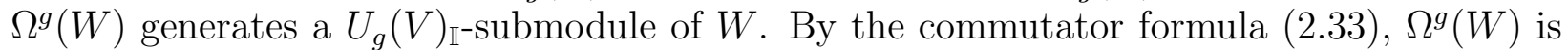
invariant under the action of $v($ wt $v+a-1)$ for weight homogeneous $v \in V^{[a+\mathbb{Z}]}, a \in P(V) \cap \mathbb{I}$. In particular, the restriction of $v($ wt $v+a-1)$ to $\Omega^{g}(W)$ gives us a linear operator $o_{W}(v)$ on $\Omega^{g}(W)$. Also by the commutator formula (2.33), $u_{1}\left(m_{1}\right) \cdots u_{k}\left(m_{k}\right) w=0$ for homogeneous $u_{i} \in V^{\left[a_{i}\right]}, w \in \Omega^{g}(W)$ and $m_{i} \in a_{i}+\mathbb{Z}$ satisfying $\Re\left(\right.$ wt $u_{1}-m_{1}-1+\cdots+$ wt $u_{k}-m_{k}-1+$ wt $w)<0$. Thus $N\left(U_{g}(V)_{\mathbb{I}}\right)$ acts on $\Omega^{g}(W)$ as 0 . In particular, $\Omega^{g}(W)$ is a $U_{g}(V)_{\mathbb{I}}$-module and moreover $\Omega^{g}(W)$ is a $Z_{g}(V)$-module with the action of $Z_{g}(V)$ given by $\left[o_{g}(v)\right] w=o_{W}(v) w$ for $v \in V$ and $w \in \Omega^{g}(W)$. Note that the weight of the operator $v$ (wt $v+a-1$ ) for weight homogeneous $v \in V^{[a+\mathbb{Z}]}$ where $a \in P(V) \cap \mathbb{I}$ is $-a \in \mathbb{I}$. Thus we have:

Proposition 5.2 The action of $Z_{g}(V)$ on $\Omega^{g}(W)$ given by $\left[o_{g}(v)\right] w=o_{W}(v)$ w gives $\Omega^{g}(W) a$ g-graded $Z_{g}(V)$-module structure. When $W$ is a lower bounded $g$-twisted $V$-module, $\Omega^{g}(W)$ is a lower bounded $Z_{g}(V)$-module. In this case, $\coprod_{n \in r+\mathbb{I}}\left(\Omega^{g}(W)\right)_{[n]}$ for $r \in \mathbb{R}$ are $Z_{g}(V)$ submodules of $\Omega^{g}(W)$.

Theorem 5.3 The functor $\Omega_{g}$ from the category of $\overline{\mathbb{C}}_{+}$-graded weak g-twisted $V$-modules to the category of g-graded $Z_{g}(V)$-modules given by $W \mapsto \Omega_{g}(W)$ has a right inverse, that is, there exists a functor $H_{g}$ from the category of g-graded $Z_{g}(V)$-modules to the category of $\overline{\mathbb{C}}_{+}$-graded weak g-twisted $V$-modules such that $\Omega_{g} \circ H_{g}=1$, where 1 is the identity functors on the category of g-graded $Z_{g}(V)$-modules. Moreover we can find such $H_{g}$ such that for any $\overline{\mathbb{C}}_{+}$-graded weak g-twisted $V$-module $W$, there exists a natural surjective homomorphism of $\overline{\mathbb{C}}_{+}$-graded weak g-twisted $V$-modules from $H_{g}\left(\Omega_{g}(W)\right)$ to the $\overline{\mathbb{C}}_{+}$-graded weak g-twisted $V$ submodule of $W$ generated by $\Omega_{g}(W)$. The restriction of $\Omega_{g}$ to the category of lower bounded $g$-twisted $V$-modules (or (grading-restricted generalized) g-twisted $V$-modules) is a functor 
from this subcategory to the category of lower bounded $Z_{g}(V)$-modules (or grading-restricted $Z_{g}(V)$-modules). The restrictions of $H_{g}$ to the category of lower bounded $Z_{g}(V)$-modules (or grading-restricted $Z_{g}(V)$-modules) is the right inverse of the restriction of $\Omega_{g}$ to the category of lower bounded $g$-twisted $V$-modules (or (grading-restricted generalized) g-twisted $V$-modules) such that for any object $W$ in the category, there exists a natural surjective homomorphism in the category from $H_{g}\left(\Omega_{g}(W)\right)$ to the lower bounded g-twisted $V$-submodule (or the (grading-restricted generalized) g-twisted $V$-submodule) of $W$ generated by $\Omega_{g}(W)$.

Proof. Let $M=\coprod_{\alpha \in \mathbb{C} / \mathbb{Z}} M^{[\alpha]}$ be a $g$-graded $Z_{g}(V)$-module. We now construct a $U_{g}(V)$ module $H_{g}(M)=\coprod_{n \in \overline{\mathbb{C}}_{+}, \alpha \in \mathbb{C} / \mathbb{Z}} H_{g}(M)_{n}^{[\alpha]}$ equipped with gradings by $\overline{\mathbb{C}}_{+}$and $\mathbb{C} / \mathbb{Z}$ and an action of $g$ on $W$ satisfying Conditions 1 to 3 in Theorem 3.5. Then by Theorem 3.5, we see that $H_{g}(M)$ is a $\overline{\mathbb{C}}_{+}$-graded weak $g$-twisted $V$-module.

From the definition of $U_{g}(V), U_{g}(V)_{-}=\coprod_{a \in \tilde{P}(V), i \in \mathbb{N}, \Re(a+i)>0} U_{g}(V)_{-a-i}$ is a subalgebra of $U_{g}(V)$. Since $M$ is a $Z_{g}(V)$-module, it is also a $U_{g}(V)_{\mathbb{I}}$-module. We define the action of $U_{g}(V)_{-}$on $M$ to be 0 . Then $M$ becomes a $U_{g}(V)_{-} \oplus U_{g}(V)_{\mathbb{I}}$-module. Let $H_{g}(M)=$ $U_{g}(V) \otimes_{U_{g}(V)_{-} \oplus U_{g}(V)_{\mathbb{I}}} M$. Then $H_{g}(M)$ is a $U_{g}(V)$-module. By Proposition 3.4, elements of $U_{g}(V)_{k}$ are of the form $u_{1}+u_{2}$ where $u_{1} \in \pi_{U_{g}(V)} T(L(V, g))_{k}$, and $u_{2}$ is in the topological completion of

$$
\coprod_{a \in \tilde{P}(V), i \in \mathbb{Z}_{+}}\left(\pi_{U_{g}(V)} T(L(V, g))_{k+a+i}\right)\left(\pi_{U_{g}(V)} T(L(V, g))_{-a-i}\right)
$$

Since $\left(\pi_{U_{g}(V)} T(L(V, g))_{-a-i}\right) \subset U_{g}(V)_{-a-i} \subset U_{g}(V)_{-}$for $a \in \tilde{P}(V)$ and $i \in \mathbb{N}$ satisfying $\Re(a+i)>0$, it acts on $M$ as 0 and thus $u_{2} w=0$ for $w \in M$. Since $u_{1} \in \pi_{U_{g}(V)} T(L(V, g))_{k}$, it is a linear combination of elements of the form $u_{1}\left(m_{1}\right) \cdots u_{k}\left(m_{k}\right)$ for $u_{i} \in V^{\left[a_{i}+\mathbb{Z}\right]}, a_{i} \in P(V)$, $m_{i} \in a_{i}+\mathbb{Z}$. Thus elements of $H_{g}(M)$ are finite linear combinations of elements of the form $u_{1}\left(m_{1}\right) \cdots u_{k}\left(m_{k}\right) w$ for $u_{i} \in V^{\left[a_{i}+\mathbb{Z}\right]}, a_{i} \in P(V), m_{i} \in a_{i}+\mathbb{Z}$ and $w \in M$. Using the commutator formula repeatedly and the actions of $U_{g}(V)_{-}$and $U_{g}(V)_{\mathbb{I}}$ on $M$, we see that $H_{g}(M)$ is spanned by elements of the same form for homogeneous $u_{i} \in V^{\left[a_{i}+\mathbb{Z}\right]}, a_{i} \in P(V)$, $m_{i} \in a_{i}+\mathbb{Z}$ and $w \in M$, satisfying $\Re\left(\right.$ wt $\left.u_{i}-m_{i}-1\right)>0$.

For an element of the form $u_{1}\left(m_{1}\right) \cdots u_{k}\left(m_{k}\right)$ for weight homogeneous $u_{i} \in V^{\left[a_{i}+\mathbb{Z}\right]}, a_{i} \in$ $P(V), m_{i} \in a_{i}+\mathbb{Z}$, we define its $\overline{\mathbb{C}}_{+}$-degree to be wt $u_{1}-m_{1}-1+\cdots+$ wt $u_{k}-m_{k}-1$. This gives a $\overline{\mathbb{C}}_{+}$-grading on $H_{g}(M)$. The actions of $g$ on $U_{g}(V)$ and $M$ also induce an action of $g$ on $H_{g}(M)$. Moreover, the $\mathbb{C} / \mathbb{Z}$-gradings by generalized eigenspaces for the actions of $g$ on $U_{g}(V)$ and $M$ give a $\mathbb{C} / \mathbb{Z}$-grading by generalized eigenspaces for the action of $g$ on $H_{g}(M)$. The $\mathbb{C} / \mathbb{Z}$-grading of $H_{g}(M)$ can also be given explicitly by defining the $\mathbb{C} / \mathbb{Z}$ degree of $u_{1}\left(m_{1}\right) \cdots u_{k}\left(m_{k}\right) w$ to be $\sum_{i=1}^{k} a_{i}+b+\mathbb{Z}$ when $w \in W^{[b+\mathbb{Z}]}$.

Condition 1 in Theorem 3.5 is satisfied by $H_{g}(M)$ because of the definition of the action of $g$ on $H_{g}(M)$. Condition 2 in Theorem 3.5 is also satisfied by $H_{g}(M)$ because $u(a+$ $m)\left(u_{1}\left(m_{1}\right) \cdots u_{k}\left(m_{k}\right) w\right)=0$ when $m>\Re\left(\right.$ wt $u-a-1+\sum_{i=1}^{k}\left(\right.$ wt $\left.\left.u_{i}-m_{i}-1\right)\right)$. It is clear that $u_{n}$ changes the $\overline{\mathbb{C}}_{+}$-degree by wt $u-n-1$ and the degree 0 homogeneous subspace of $H_{g}(M)$ is $M$ which is also the subspace of $H_{g}(M)$ annihilated by $U_{g}(V)_{-}$. Moreover, $H_{g}(M)$ 
is a direct sum of generalized eigenspaces for the action of $g$ on $H_{g}(M)$. Thus Condition 3 is satisfied.

Since our construction is natural, we obtain a functor $H_{g}$ from the category of $Z_{g}(V)$ modules to the category of $\overline{\mathbb{C}}_{+}$-graded weak $g$-twisted $V$-modules.

By definition, $M \subset \Omega_{g}\left(H_{g}(M)\right)$. Since $H_{g}(M)$ is spanned by elements of the form $u_{1}\left(m_{1}\right) \cdots u_{k}\left(m_{k}\right) w$ for homogeneous $u_{i} \in V^{\left[a_{i}+\mathbb{Z}\right]}, a_{i} \in P(V), m_{i} \in a_{i}+\mathbb{Z}$ and $w \in M$ satisfying wt $u_{i}-m_{i}-1>0$, we see that $\Omega_{g}\left(H_{g}(M)\right)=M$. Thus we have $\Omega_{g} \circ H_{g}=1$.

Now let $W$ be a $\overline{\mathbb{C}}_{+}$-graded weak $g$-twisted $V$-module. Then the $\overline{\mathbb{C}}_{+}$-graded weak $g$-twisted $V$-submodule of $W$ generated by $\Omega_{g}(W)$ is spanned by elements of the form $u_{1}\left(m_{1}\right) \cdots u_{k}\left(m_{k}\right) w$ for $u_{i} \in V^{\left[a_{i}+\mathbb{Z}\right]}, a_{i} \in P(V), m_{i} \in a_{i}+\mathbb{Z}$ and $w \in \Omega_{g}(W)$. Define a linear map from $H_{g}\left(\Omega_{g}(W)\right)$ to this submodule of $W$ by sending the elements of the same form in $H_{g}\left(\Omega_{g}(W)\right)$ to these corresponding elements in this submodule. This linear map is well defined because the only relations among these elements in $H_{g}\left(\Omega_{g}(W)\right)$ are given by the commutator formula, the weak associativity, the $L(-1)$-derivative property, the properties for the vacuum and the Virasoro relations and these relations are all satisfied by the corresponding elements in the submodule of $W$. By definition, this linear map is a surjective module map from $H_{g}\left(\Omega_{g}(W)\right)$ to this submodule of $W$.

The other conclusions follows immediately.

We now discuss $\tilde{A}_{g}(V)$ - and $A_{g}(V)$-modules and $\overline{\mathbb{C}}_{+}$-graded weak $g$-twisted $V$-modules. As in the case of $Z_{g}(V)$-modules, we also discuss only $\tilde{A}_{g}(V)$ - and $A_{g}(V)$-modules with actions of $g$ and are direct sums of generalized eigenspaces of the actions of $g$. As in the case of $Z_{g}(V)$-modules, we are also interested in $g$-graded $\tilde{A}_{g}(V)$-modules with another grading given by the generalized eigenspaces of $\omega+\tilde{O}_{g}(V) \in \tilde{A}_{g}(V)$.

Definition 5.4 Let $M$ be an $\tilde{A}_{g}(V)$ - or $A_{g}(V)$-module. If $M=\coprod_{\alpha \in \mathbb{C} / \mathbb{Z}} M^{[\alpha]}$ where $M^{[\alpha]}$ for $\alpha \in \mathbb{C} / \mathbb{Z}$ are the generalized eigenspaces of the action of $g$, then we call $M$ a $g$-graded $\tilde{A}_{g}(V)$ and $A_{g}(V)$-module. Let $M=\coprod_{n \in \mathbb{C}, \alpha \in \mathbb{C} / \mathbb{Z}} M_{[n]}^{[\alpha]}$ be an $\tilde{A}_{g}(V)$-module with double gradings by $\mathbb{C}$ and by $\mathbb{C} / \mathbb{Z}$ (or equivalently, by a subset of $[0,1)+\mathbb{I}$ ). If $M_{[n]}=\coprod_{\alpha \in \mathbb{C} / \mathbb{Z}} M_{[n]}^{[\alpha]}$ for $n \in \mathbb{C}$ are generalized eigenspaces of the action of $\omega+\tilde{O}_{g}(V)$ on $M$ with eigenvalues $n$ (called weights) and the $\mathbb{C} / \mathbb{Z}$-grading is given by the generalized eigenspaces of an action of $g$ compatible with the $\tilde{A}_{g}(V)$-module structure, then we call $M$ a doubly-graded $\tilde{A}_{g}(V)$-module or simply a graded $\tilde{A}_{g}(V)$-module. Let $M=\coprod_{n \in \mathbb{C}, \alpha \in \mathbb{C} / \mathbb{Z}} M_{[n]}^{[\alpha]}$ be a graded $\tilde{A}_{g}(V)$-module, if for each $n \in \mathbb{C}$ and each $\alpha \in \mathbb{C} / \mathbb{Z}, M_{[n+l]}^{[\alpha]}=0$ for sufficiently negative real number $l$, we call $M$ a lower bounded $\tilde{A}_{g}(V)$-module. A lower bounded $\tilde{A}_{g}(V)$-module $M=\coprod_{n \in \mathbb{C}, \alpha \in \mathbb{C} / \mathbb{Z}} M_{[n]}^{[\alpha]}$ is called grading restricted if $\operatorname{dim} M_{[n]}^{[\alpha]}<\infty$ for $n \in \mathbb{C}, \alpha \in \mathbb{C} / \mathbb{Z}$. The notions of graded $A_{g}(V)$ module, lower bounded $A_{g}(V)$-module and grading-restricted $A_{g}(V)$-module are defined in the same way.

Note that in particular, $\tilde{A}_{g}(V)\left(A_{g}(V)\right)$ itself is a $g$-graded $\tilde{A}_{g}(V)$-module $\left(A_{g}(V)\right.$-module). 
Let $W=\coprod_{n \in \overline{\mathbb{C}}_{+}, \alpha \in \mathbb{C} / \mathbb{Z}} W_{[n]}^{[\alpha]}$ be a $\overline{\mathbb{C}}_{+}$-graded weak $g$-twisted $V$-module. Define two linear maps $o_{W}$ and $\rho_{W}=o_{W} \circ \mathcal{U}(1)$ from $V$ to End $\Omega^{g}(W)$ by $v \mapsto o_{W}(v)$ and $v \mapsto o_{W}(\mathcal{U}(1) v)$, respectively, for $v \in V$, where

$$
o_{W}(v) w=\operatorname{Res}_{x} x^{a-1} Y_{0}\left(x^{L(0)} v, x\right) w=v(\text { wt } v+a-1) w
$$

for $a \in P(V) \cap \mathbb{I}$, weight homogeneous $v \in V^{[a+\mathbb{Z}]}, w \in \Omega^{g}(W)$ and

$$
o_{W}(v) w=0
$$

when $a \notin \mathbb{I}, v \in V^{[a+\mathbb{Z}]}, w \in \Omega^{g}(W)$. By definition, $o_{W}$ and $\rho_{W}$ are determined by their restrictions to $\coprod_{a \in P(V) \cap \mathbb{I}} V^{[a+\mathbb{Z}]}$.

We have the following:

Theorem 5.5 The spaces $O_{g}(V)$ and $\tilde{O}_{g}(V)$ are in the kernels of $o_{W}$ and $\rho_{W}$, respectively, and the maps given by $u+O_{g}(V) \mapsto o_{W}(u)$ and $u+\tilde{O}_{g}(V) \mapsto \rho_{W}(u)$ for $u \in V$ give $\Omega_{g}(W)$ g-graded $A_{g}(V)$ - and $\tilde{A}_{g}(V)$-module structures, respectively. When $W$ is a lower bounded $g$-twisted $V$-module, $\Omega^{g}(W)$ is a lower bounded $A_{g}(V)$ - or $\tilde{A}_{g}(V)$-module. In particular, $\coprod_{n \in r+\mathbb{I}}\left(\Omega^{g}(W)\right)_{[n]}$ for $r \in \mathbb{R}$ are $A_{g}(V)$ - or $\tilde{A}_{g}(V)$-submodules of $\Omega^{g}(W)$.

Proof. As in the proofs of Theorem 4.1 and Corollary 4.2, the proofs of the first statement for $\tilde{O}_{g}(V)$ and $\tilde{A}_{g}(V)$ are the same as the corresponding proofs of the first statement in Proposition 6.4 in [H2] except for the use of (2.14) and for the adjustments involving $a \in$ $P(V)$. The proofs for $O_{g}(V)$ and $A_{g}(V)$ can be obtained either from those for $\tilde{O}_{g}(V)$ and $\tilde{A}_{g}(V)$ using the map $\mathcal{U}(1)$ or by directly generalizing the proof in [Z] and [DLM1].

Here we give only the proof of the statement that

$$
\rho_{W}\left(\operatorname{Res}_{x_{0}} \frac{2 \pi \sqrt{-1} e^{2 \pi \sqrt{-1} a x_{0}}}{\left(e^{2 \pi \sqrt{-1}} x_{0}-1\right)^{n}} Y\left(e^{2 \pi \sqrt{-1} x_{0} \mathcal{N}} u, x_{0}\right) v\right)=0
$$

for $u \in V^{[a+\mathbb{Z}]}, a \in P(V), 0<\Re(a)<1$, and $v \in V^{[b+\mathbb{Z}]}$.

If $a+b-1 \notin \mathbb{I}, Y\left(e^{2 \pi \sqrt{-1} x_{0} \mathcal{N}} u, x_{0}\right) v \in V^{[a+b+\mathbb{Z}]}\left[\left[x, x^{-1}\right]\right]$ where $a+b+\mathbb{Z} \notin \mathbb{I}+\mathbb{Z}$. Then by definition,

$$
\rho_{W}\left(Y\left(e^{2 \pi \sqrt{-1} x_{0} \mathcal{N}} u, x_{0}\right) v\right)=0
$$

and thus (5.8) holds. So we need only consider the case $a+b-1 \in \mathbb{I}$. In this case, for $n \in \mathbb{Z}_{+}$and $w \in \Omega_{g}(W)$, using the definitions, (1.5) in [H2], the $L(0)$-conjugation formula, (2.16), the basic property of the formal delta-function and the Jacobi identity (2.29) for $Y_{0}$, we have

$$
\begin{aligned}
\rho_{W} & \left(\operatorname{Res}_{x_{0}} \frac{2 \pi \sqrt{-1} e^{2 \pi \sqrt{-1} a x_{0}}}{\left(e^{2 \pi \sqrt{-1} x_{0}}-1\right)^{n}} Y\left(e^{2 \pi \sqrt{-1} x_{0} \mathcal{N}} u, x_{0}\right) v\right) w \\
\quad & \operatorname{Res}_{x_{2}} \frac{1}{x_{2}^{(1-a-b)+1}} \operatorname{Res}_{x_{0}} \frac{2 \pi \sqrt{-1} e^{2 \pi \sqrt{-1}} a x_{0}}{\left(e^{2 \pi \sqrt{-1} x_{0}}-1\right)^{n}} Y_{0}\left(\mathcal{U}\left(x_{2}\right) Y\left(e^{2 \pi \sqrt{-1} x_{0} \mathcal{N}} u, x_{0}\right) v, x_{2}\right) w
\end{aligned}
$$




$$
\begin{aligned}
& =\operatorname{Res}_{x_{2}} \operatorname{Res}_{x_{0}} \frac{2 \pi \sqrt{-1} e^{2 \pi \sqrt{-1} a x_{0}}}{x_{2}^{2-a-b}\left(e^{2 \pi \sqrt{-1} x_{0}}-1\right)^{n}} . \\
& \cdot Y_{0}\left(x_{2}^{L(0)} Y\left(\mathcal{U}\left(e^{2 \pi \sqrt{-1} x_{0}}\right) e^{2 \pi \sqrt{-1} x_{0} \mathcal{N}} u, e^{2 \pi \sqrt{-1} x_{0}}-1\right) \mathcal{U}(1) v, x_{2}\right) w \\
& =\operatorname{Res}_{x_{2}} \operatorname{Res}_{x_{0}} \frac{2 \pi \sqrt{-1} e^{2 \pi \sqrt{-1} a x_{0}}}{x_{2}^{2-a-b}\left(e^{2 \pi \sqrt{-1} x_{0}}-1\right)^{n}} . \\
& \cdot Y_{0}\left(Y\left(x_{2}^{L(0)} \mathcal{U}\left(e^{2 \pi \sqrt{-1} x_{0}}\right) e^{2 \pi \sqrt{-1} x_{0} \mathcal{N}} u, x_{2}\left(e^{2 \pi \sqrt{-1} x_{0}}-1\right)\right) x_{2}^{L(0)} \mathcal{U}(1) v, x_{2}\right) w \\
& =\operatorname{Res}_{x_{2}} \operatorname{Res}_{x_{0}} \frac{2 \pi \sqrt{-1} e^{2 \pi \sqrt{-1} a x_{0}}}{x_{2}^{2-a-b}\left(e^{2 \pi \sqrt{-1} x_{0}}-1\right)^{n}} . \\
& \cdot Y_{0}\left(Y\left(e^{2 \pi \sqrt{-1} x_{0} \mathcal{N}} x_{2}^{L(0)} \mathcal{U}\left(e^{2 \pi \sqrt{-1} x_{0}}\right) u, x_{2}\left(e^{2 \pi \sqrt{-1} x_{0}}-1\right)\right) x_{2}^{L(0)} \mathcal{U}(1) v, x_{2}\right) w \\
& =\operatorname{Res}_{x_{2}} \operatorname{Res}_{y_{0}}\left(1+\frac{y_{0}}{x_{2}}\right)^{a-1} \frac{1}{x_{2}^{3-a-b-n} y_{0}^{n}} . \\
& \cdot Y_{0}\left(Y\left(\left(1+\frac{y_{0}}{x_{2}}\right)^{\mathcal{N}} \mathcal{U}\left(x_{2}+y_{0}\right) u, y_{0}\right) \mathcal{U}\left(x_{2}\right) v, x_{2}\right) w \\
& =\operatorname{Res}_{x_{2}} \operatorname{Res}_{y_{0}} \operatorname{Res}_{x_{1}} x_{1}^{a-1} x_{1}^{-1} \delta\left(\frac{x_{2}+y_{0}}{x_{1}}\right)\left(\frac{x_{2}+y_{0}}{x_{1}}\right)^{a} \frac{x_{2}^{b-2+n}}{y_{0}^{n}} \text {. } \\
& \cdot Y_{0}\left(Y\left(\left(1+\frac{y_{0}}{x_{2}}\right)^{\mathcal{N}} \mathcal{U}\left(x_{2}+y_{0}\right) u, y_{0}\right) \mathcal{U}\left(x_{2}\right) v, x_{2}\right) w \\
& =\operatorname{Res}_{x_{2}} \operatorname{Res}_{y_{0}} \operatorname{Res}_{x_{1}} x_{1}^{a-1} x_{1}^{-1} \delta\left(\frac{x_{2}+y_{0}}{x_{1}}\right)\left(\frac{x_{2}+y_{0}}{x_{1}}\right)^{a} \frac{x_{2}^{b-2+n}}{y_{0}^{n}} \text {. } \\
& \cdot Y_{0}\left(Y\left(\left(1+\frac{y_{0}}{x_{2}}\right)^{\mathcal{N}} \mathcal{U}\left(x_{1}\right) u, y_{0}\right) \mathcal{U}\left(x_{2}\right) v, x_{2}\right) w \\
& =\operatorname{Res}_{x_{2}} \operatorname{Res}_{y_{0}} \operatorname{Res}_{x_{1}} \frac{x_{1}^{a-1} x_{2}^{b-2+n}}{y_{0}^{n}} y_{0}^{-1} \delta\left(\frac{x_{1}-x_{2}}{y_{0}}\right) \text {. } \\
& \cdot Y_{0}\left(\mathcal{U}\left(x_{2}+y_{0}\right) u, x_{1}\right) Y_{0}\left(\mathcal{U}\left(x_{2}\right) v, x_{2}\right) w \\
& -\operatorname{Res}_{x_{2}} \operatorname{Res}_{y_{0}} \operatorname{Res}_{x_{1}} \frac{x_{1}^{a-1} x_{2}^{b-2+n}}{y_{0}^{n}} y_{0}^{-1} \delta\left(\frac{x_{2}-x_{1}}{-y_{0}}\right) \text {. } \\
& \cdot Y_{0}\left(\mathcal{U}\left(x_{2}\right) v, x_{2}\right) Y_{0}\left(\mathcal{U}\left(x_{2}+y_{0}\right) u, x_{1}\right) w \\
& =\operatorname{Res}_{x_{2}} \operatorname{Res}_{x_{1}} \frac{x_{1}^{a-1} x_{2}^{b-2+n}}{\left(x_{1}-x_{2}\right)^{n}} Y_{0}\left(\mathcal{U}\left(x_{1}\right) u, x_{1}\right) Y_{0}\left(\mathcal{U}\left(x_{2}\right) v, x_{2}\right) w \\
& -\operatorname{Res}_{x_{2}} \operatorname{Res}_{x_{1}} \frac{x_{1}^{a-1} x_{2}^{b-2+n}}{\left(-x_{2}+x_{1}\right)^{n}} Y_{0}\left(\mathcal{U}\left(x_{2}\right) v, x_{2}\right) Y_{0}\left(\mathcal{U}\left(x_{1}\right) u, x_{1}\right) w .
\end{aligned}
$$

Since $w \in \Omega_{g}(W)$, the right-hand side of (5.9) is 0 .

Since the weight of the operator $\rho_{W}(u)$ is $\mathbb{I}, \coprod_{n \in r+\mathbb{I}}\left(\Omega^{g}(W)\right)_{[n]}$ for $r \in \mathbb{R}$ are invariant under $\rho_{W}(u)$ and thus are $\tilde{A}(V)$-submodules of $\Omega^{g}(W)$. The compatibility with the action of $g$ clear. 
Theorem 5.6 The functor $\Omega_{g}$ from the category of $\overline{\mathbb{C}}_{+}$-graded weak g-twisted $V$-modules to the category of g-graded $A_{g}(V)$-modules or $\tilde{A}_{g}(V)$-modules given by $W \mapsto \Omega_{g}(W)$ has a right inverse, that is, there exist functors $S_{g}$ and $\tilde{S}_{g}$ from the categories of $g$-graded $A_{g}(V)$-modules and $\tilde{A}_{g}(V)$-modules, respectively, to the category of $\overline{\mathbb{C}}_{+}$-graded weak $g$-twisted $V$-modules such that $\Omega_{g} \circ S_{g}=1$ and $\Omega_{g} \circ \tilde{S}_{g}=\tilde{1}$, where 1 and $\tilde{1}$ are the identity functors on the categories of $A_{g}(V)$-modules and $\tilde{A}_{g}(V)$-modules, respectively. Moreover we can find such $S_{g}$ and $\tilde{S}_{g}$ such that for any $\overline{\mathbb{C}}_{+}$-graded weak g-twisted $V$-module $W$, there exists a natural surjective homomorphism of $\overline{\mathbb{C}}_{+}$-graded weak g-twisted $V$-modules from $S_{g}\left(\Omega_{g}(W)\right)$ or $\tilde{S}_{g}\left(\Omega_{g}(W)\right)$ to the $\overline{\mathbb{C}}_{+}$-graded weak g-twisted $V$-submodule of $W$ generated by $\Omega_{g}(W)$. The restriction of $\Omega_{g}$ to the category of lower bounded $g$-twisted $V$-modules (or (grading-restricted) g-twisted $V$-mdoules) is a functor from this subcategory to the category of lower bounded (or gradingrestricted) $A_{g}(V)$-modules or $\tilde{A}_{g}(V)$-modules. The restrictions of $S_{g}$ and $\tilde{S}_{g}$ to the categories of lower bounded (or grading-restricted) $A_{g}(V)$-modules and $\tilde{A}_{g}(V)$-modules, respectively, are right inverses of the restriction of $\Omega_{g}$ to the categories of lower bounded (or grading-restricted) $g$-twisted $V$-modules such that for any lower bounded (or grading-restricted) g-twisted $V$ module $W$, there exists a natural surjective homomorphism of such g-twisted $V$-modules from $S_{g}\left(\Omega_{g}(W)\right)$ or $\tilde{S}_{g}\left(\Omega_{g}(W)\right)$ to the lower bounded (or grading-restricted) g-twisted $V$-submodule of $W$ generated by $\Omega_{g}(W)$.

Proof. We prove only the results for $\tilde{A}_{g}(V)$. The proofs of the results for $A_{g}(V)$ can be derived from the results for $\tilde{A}_{g}(V)$. The proofs of these results are the same as the proofs of the second and third statements of Proposition 6.4 in [H2] except that instead of expressions corresponding to the commutator formula, the $L(-1)$-derivative property and the associator formula for $V$-modules, here we use the expressions corresponding to (2.31), (2.18) and (2.35).

We need to construct a $\overline{\mathbb{C}}_{+}$-graded weak $g$-twisted $V$-module from a $g$-graded $\tilde{A}_{g}(V)$ module. Recall the space $L(V, g)$, the tensor algebra $T(L(V, g))$ and the notations we have used in Section 3.

Let $M=\coprod_{\alpha \in \mathbb{C} / \mathbb{Z}} M^{[\alpha]}$ be a $g$-graded $\tilde{A}_{g}(V)$-module and let $\rho: \tilde{A}_{g}(V) \rightarrow$ End $M$ be the map giving the representation of $\tilde{A}_{g}(V)$ on $M$. Consider $T(L(V, g)) \otimes M$. Again for simplicity we shall omit the tensor product sign. So $T(L(V, g)) \otimes M$ is spanned by elements of the form $u_{1}\left(m_{1}\right) \cdots u_{k}\left(m_{k}\right) w$ for $u_{i} \in V^{\left[a_{i}+\mathbb{Z}\right]}, m_{i} \in a_{i}+\mathbb{Z}, a_{i} \in P(V), i=1, \ldots, k$, and $w \in M_{[n]}^{[b+\mathbb{Z}]}$, $b \in[0,1)+\mathbb{I}, n \in \mathbb{C}$. For $u \in V^{[a+\mathbb{Z}]}, m \in a+\mathbb{Z}$ where $a \in P(V), u(m)$ acts from the left on $T(L(V, g)) \otimes M$. In the case that $u_{i}$ are homogeneous with respect to the grading by weights, we define the $\overline{\mathbb{C}}_{+}$-degree of $u_{1}\left(m_{1}\right) \cdots u_{k}\left(m_{k}\right) w$ to be $\left(\right.$ wt $\left.u_{1}-m_{1}-1\right)+\cdots+\left(\right.$ wt $\left.u_{k}-m_{k}-1\right)$ and its $\mathbb{C} / \mathbb{Z}$ degree by $a_{1}+\cdots+a_{k}+b+\mathbb{Z}$. For any $u \in V^{[a+\mathbb{Z}]}$, let

$$
Y_{0}^{t}(u, x): T(L(V, g)) \otimes M \rightarrow T(L(V, g))\left[\left[x, x^{-1}\right]\right]
$$

be defined by

$$
Y_{0}^{t}(u, x)=\sum_{m \in a+\mathbb{Z}} u(m) x^{-m-1} .
$$

For a homogeneous element $u \in V^{[a+\mathbb{Z}]}, a \in P(V) \cap \mathbb{I}$, let $o_{t}(u)=u($ wt $u+a-1)$. Using linearity, we extend $o_{t}(u)$ to inhomogeneous $u \in \coprod_{a \in P(V) \cap \mathbb{I}} V^{[a+\mathbb{Z}]}$. 
Let $\mathcal{I}$ be the graded $T(L(V, g))$-submodule of $T(L(V, g)) \otimes M$ generated by elements of the forms $u(m) w\left(u \in V^{[a+\mathbb{Z}]}, a \in P(V), m \in a+\mathbb{Z}\right.$, wt $\left.u-m-1<0, w \in M\right)$, $o_{t}(\mathcal{U}(1) u) w-\rho(u+\tilde{O}(V)) w\left(u \in \coprod_{a \in P(V) \cap \mathbb{I}} V^{[a+\mathbb{Z}]}, w \in M\right)$ and the coefficients in $x_{1}$ and $x_{2}$ of

$$
\begin{aligned}
& Y_{0}^{t}\left(u, x_{1}\right) Y_{0}^{t}\left(v, x_{2}\right) w-Y_{0}^{t}\left(v, x_{2}\right) Y_{0}^{t}\left(u, x_{1}\right) w \\
& \quad-\operatorname{Res}_{x_{0}} x_{1}^{-1} \delta\left(\frac{x_{2}+x_{0}}{x_{1}}\right)\left(\frac{x_{2}+x_{0}}{x_{1}}\right)^{a} Y_{0}^{t}\left(Y\left(\left(1+\frac{x_{0}}{x_{2}}\right)^{\mathcal{N}} u, x_{0}\right) v, x_{2}\right) w
\end{aligned}
$$

$\left(u \in V^{[a+\mathbb{Z}]}, v \in V, a \in P(V)\right.$ and $\left.w \in T(L(V, g)) \otimes M\right)$. Note that as in the proof of Proposition 6.4 in [H2], the coefficients of the formal expression above are indeed in $T(L(V, g)) \otimes M$. Let $S_{g}^{1}(M)=(T(L(V, g)) \otimes M) / \mathcal{I}$. Then $S_{g}^{1}(M)$ is also a graded $T(L(V, g))$ module. In fact, by the definition of $\mathcal{I}$, we see that $S_{g}^{1}(M)$ is spanned by elements of the form $u_{1}\left(m_{1}\right) \cdots u_{k}\left(m_{k}\right) w+\mathcal{I}$ for $k \in \mathbb{N}$, homogeneous $u_{i} \in V^{\left[a_{i}+\mathbb{Z}\right]}, m_{i} \in a_{i}+\mathbb{Z}, a_{i} \in P(V)$, $i=1, \ldots, k$, and $w \in M^{[b+\mathbb{Z}]}, b \in[0,1)+\mathbb{I}$, satisfying $\Re\left(\right.$ wt $\left.u_{1}-m_{1}-1\right) \geq \cdots \geq \Re\left(\right.$ wt $u_{k}-$ $\left.m_{k}-1\right)>0$. In particular, $S_{g}^{1}(M)$ is doubly graded by $\overline{\mathbb{C}}_{+}$and $\mathbb{C} / \mathbb{Z}$. Thus for homogeneous $u \in V$ and $w \in S_{g}^{1}(M), u(m) w=0$ when the real part of $m$ is sufficiently large. Moreover, $S_{g}^{1}(M)=S_{g}^{1}(M)_{+} \oplus S_{g}^{1}(M)_{0}$, where $S_{g}^{1}(M)_{+}$is the subspace of $S_{g}^{1}(M)$ spanned by elements of the form above for $k \in \mathbb{Z}_{+}$and $S_{g}^{1}(M)_{0}$ is spanned by the elements of the form $w+\mathcal{I}$ for $w \in M$.

We show that $S_{g}^{1}(M)_{0}$ is linearly isomorphic to $M$. We need only show that if $\sum_{i=1}^{k} \rho\left(u_{1}^{i}+\right.$ $\tilde{O}(V)) \cdots \rho\left(u_{k}^{i}+\tilde{O}(V)\right) w \neq 0$ in $M$, then $\sum_{i=1}^{k} \rho\left(u_{1}^{i}+\tilde{O}(V)\right) \cdots \rho\left(u_{k}^{i}+\tilde{O}(V)\right) w+\mathcal{I} \neq 0$ in $S_{g}^{1}(M)_{0}$, or equivalently, $\sum_{i=1}^{k} o_{t}\left(\mathcal{U}(1) u_{1}^{i}\right) \cdots o_{t}\left(\mathcal{U}(1) u_{k}^{i}\right) w \notin \mathcal{I}$. Assume that this is not true, that is, $\sum_{i=1}^{k} o_{t}\left(\mathcal{U}(1) u_{1}^{i}\right) \cdots o_{t}\left(\mathcal{U}(1) u_{k}^{i}\right) w \in \mathcal{I}$. Since $\sum_{i=1}^{k} \rho\left(u_{1}^{i}+\tilde{O}(V)\right) \cdots \rho\left(u_{k}^{i}+\tilde{O}(V)\right) w \neq 0$ in $M, \sum_{i=1}^{k} o_{t}\left(\mathcal{U}(1) u_{1}^{i}\right) \cdots o_{t}\left(\mathcal{U}(1) u_{k}^{i}\right) w$ cannot be in the graded $T(L(V, g))$-submodule of $T(L(V, g)) \otimes M$ generated by elements of the forms $o_{t}(\mathcal{U}(1) u) w-\rho(u+\tilde{O}(V)) w(u \in$ $\left.\coprod_{a \in P(V) \cap \mathbb{I}} V^{[a+\mathbb{Z}]}, w \in M\right)$. Clearly, $\sum_{i=1}^{k} o_{t}\left(\mathcal{U}(1) u_{1}^{i}\right) \cdots o_{t}\left(\mathcal{U}(1) u_{k}^{i}\right) w$ is not in the graded $T(L(V, g))$-submodule of $T(L(V, g)) \otimes M$ generated by elements of the forms $u(m) w(u \in$ $V^{[a+\mathbb{Z}]}, a \in P(V), m \in a+\mathbb{Z}$, wt $\left.u-m-1<0, w \in M\right)$. Thus $\sum_{i=1}^{k} o_{t}\left(\mathcal{U}(1) u_{1}^{i}\right) \cdots o_{t}\left(\mathcal{U}(1) u_{k}^{i}\right) w$ is in fact in the the graded $T(L(V, g))$-submodule $\widetilde{\mathcal{I}}$ of $T(L(V, g)) \otimes M$ generated by the coefficients in $x_{1}$ and $x_{2}$ of (5.10) $\left(u \in V^{[a+\mathbb{Z}]}, v \in V, a \in P(V)\right.$ and $\left.w \in T(L(V, g)) \otimes M\right)$. Then by the definition of $\widetilde{\mathcal{I}}, \sum_{i=1}^{k} o_{t}\left(\mathcal{U}(1) u_{1}\right) \cdots o_{t}\left(\mathcal{U}(1) u_{k}\right)$ must be in the subalgebra of $T(L(V, g))$ spanned by products of at least one element of the form

$$
o_{t}\left(\mathcal{U}(1) v_{1}\right) o_{t}\left(\mathcal{U}(1) v_{2}\right)-o_{t}\left(\mathcal{U}(1) v_{2}\right) o_{t}\left(\mathcal{U}(1) v_{1}\right)-2 \pi \sqrt{-1} \operatorname{Res}_{x_{0}} o_{t}\left(\mathcal{U}(1) Y\left(e^{2 \pi \sqrt{-1} x\left(a_{1}+\mathcal{N}\right)} v_{1}, x_{0}\right) v_{2}\right)
$$

for $v_{j} \in V^{\left[a_{j}+\mathbb{Z}\right]}, a_{j} \in P(V) \cap \mathbb{I}$ for $j=1,2$, and elements of the form $o_{t}(\mathcal{U}(1) v)$ for $v \in V^{[a+\mathbb{Z}]}$, $a \in P(V) \cap \mathbb{I}$. So the element $\sum_{i=1}^{k} \rho\left(u_{1}^{i}+\tilde{O}(V)\right) \cdots \rho\left(u_{k}^{i}+\tilde{O}(V)\right) w$ of $M$ corresponding to $\sum_{i=1}^{k} o_{t}\left(\mathcal{U}(1) u_{1}^{i}\right) \cdots o_{t}\left(\mathcal{U}(1) u_{k}^{i}\right) w$ must be a sum of elements obtained by applying at least 
one operator of the form

$$
\begin{aligned}
\rho\left(v_{1}+\right. & \tilde{O}(V)) \rho\left(v_{2}+\tilde{O}(V)\right)-\rho\left(v_{2}+\tilde{O}(V)\right) \rho\left(v_{1}+\tilde{O}(V)\right) \\
& -2 \pi \sqrt{-1} \operatorname{Res}_{x_{0}} \rho\left(Y\left(e^{2 \pi \sqrt{-1} x\left(a_{1}+\mathcal{N}\right)} v_{1}, x_{0}\right) v_{2}+\tilde{O}(V)\right) \\
= & \rho\left(v_{1} \bullet g v_{2}-v_{2} \bullet_{g} v_{1}-2 \pi \sqrt{-1} \operatorname{Res}_{x_{0}} Y\left(e^{2 \pi \sqrt{-1} x\left(a_{1}+\mathcal{N}\right)} v_{1}, x_{0}\right) v_{2}+\tilde{O}(V)\right)
\end{aligned}
$$

and operators of the form $\rho(v)$ for $v \in V^{[a+\mathbb{Z}]}, a \in P(V) \cap \mathbb{I}$ to $w$. By (4.2),

$$
v_{1} \bullet_{g} v_{2}-v_{2} \bullet_{g} v_{1}-2 \pi \sqrt{-1} \operatorname{Res}_{x_{0}} Y\left(e^{2 \pi \sqrt{-1} x\left(a_{1}+\mathcal{N}\right)} v_{1}, x_{0}\right) v_{2}+\tilde{O}(V)=0 .
$$

Thus $\sum_{i=1}^{k} \rho\left(u_{1}^{i}+\tilde{O}(V)\right) \cdots \rho\left(u_{k}^{i}+\tilde{O}(V)\right) w=0$. We have a ontracdiction. So $S_{g}^{1}(M)_{0}$ is linearly isomorphic to $M$. We shall identify $S_{g}^{1}(M)_{0}$ with $M$. Then $S_{g}^{1}(M)=S_{g}^{1}(M)_{+} \otimes M$.

Let $\mathcal{J}$ be the $\overline{\mathbb{C}}_{+} \times \mathbb{C} / \mathbb{Z}$-graded $T(L(V, g))$-submodule of $S_{g}^{1}(M)$ generated by the coefficients in $x$ of

$$
Y_{0}^{t}(L(-1) u, x) w-\frac{d}{d x} Y_{0}^{t}(u, x) w+x^{-1} Y_{0}^{t}(\mathcal{N} u, x) w
$$

and the coefficients in $x_{0}$ and $x_{2}$ of

$$
\left(x_{0}+x_{2}\right)^{l} Y_{0}^{t}\left(u, x_{0}+x_{2}\right) Y_{0}^{t}\left(v, x_{2}\right) w-\left(x_{2}+x_{0}\right)^{l} Y_{0}^{t}\left(Y\left(\left(1+\frac{x_{0}}{x_{2}}\right)^{\mathcal{N}} u, x_{0}\right) v, x_{2}\right) w
$$

(which are indeed in $S_{g}^{1}(M)$ ) for $u \in V^{[a+\mathbb{Z}]}, v \in V, w \in S_{g}^{1}(M)$ and $l \in a+\mathbb{Z}$ satisfying $u(n) w=0$ for $n \in a+\mathbb{Z}$ such that $n-a \geq l-a$.

Let $S_{g}(M)=S_{g}^{1}(M) / \mathcal{J}$. Then $S_{g}(M)$ is also a $\overline{\mathbb{C}}_{+} \times \mathbb{C} / \mathbb{Z}$-graded $T(L(V, g))$-module. We can still use elements of $T(L(V, g)) \otimes M$ to represent elements of $S_{g}(M)$. But note that these elements now satisfy relations. We equip $S_{g}(M)$ with the vertex operator map $Y_{0}^{g}: V \otimes S_{g}(M) \rightarrow S_{g}(M)\left[\left[x, x^{-1}\right]\right]$ given by $u \otimes w \mapsto Y_{0}^{g}(u, x) w=Y_{0}^{t}(u, x) w$. As in $S_{g}^{1}(M)$, for $u \in V^{[a+\mathbb{Z}]}, m \in a+\mathbb{Z}$ and $w \in S_{g}(M)$, we also have $u(m) w=0$ when the real part of wt $u-m-1$ is sufficiently negative. Clearly $Y_{0}^{g}(\mathbf{1}, x)=1_{S_{g}(M)}$ (where $1_{S_{g}(M)}$ is the identity operator on $S_{g}(M)$ ). By definition, we know that the commutator formula, the weak associativity and the $L(-1)$-derivative property for $Y_{0}^{g}$ all hold. The $\overline{\mathbb{C}}_{+}$-grading condition is also clear. Thus $S_{g}(M)$ is a $\overline{\mathbb{C}}_{+}$-graded weak $g$-twisted $V$-module.

We still need to show that $\Omega_{g}\left(S_{g}(M)\right)=M$. To prove this fact, we need only prove that the relations given by $\mathcal{J}$ already hold in $M$. Let $\pi_{M}$ be the projection from $S_{g}^{1}(M)$ to $M$. we need only prove that

$$
\pi_{M} Y_{0}^{t}(L(-1) u, x) w-\pi_{M} \frac{d}{d x} Y_{0}^{t}(u, x) w+\pi_{M} x^{-1} Y_{0}^{t}(\mathcal{N} u, x) w=0
$$

and

$$
\left(x_{0}+x_{2}\right)^{l} \pi_{M} Y_{0}^{t}\left(u, x_{0}+x_{2}\right) Y_{0}^{t}\left(v, x_{2}\right) w=\left(x_{2}+x_{0}\right)^{l} \pi_{M} Y_{0}^{t}\left(Y\left(\left(1+\frac{x_{0}}{x_{2}}\right)^{\mathcal{N}} u, x_{0}\right) v, x_{2}\right) w
$$


hold in $S_{g}^{1}(M)$ for homogeneous $u \in V^{[a+\mathbb{Z}]}, v \in V, w \in S_{g}^{1}(M)$. Recall $l(u) \in a+\mathbb{Z}$ in Section 4 defined by wt $u-n-1<0$ (thus $u(n) w=0$ for $w \in M$ ) for $n \in a+\mathbb{Z}$ satisfying $n-a \geq l(u)-a$. We first prove these formulas for $w \in M \subset S_{g}^{1}(M)$ and $l=l(u)$.

Since $u$ is homogeneous with respect to the $\mathbb{C}$-grading (weight grading), the terms whose weights are in $\mathbb{I}$ in the series $Y_{0}^{t}(L(-1) u, x), \frac{d}{d x} Y_{0}^{t}(u, x)$ and $x^{-1} Y_{0}^{t}(\mathcal{N} u, x)$ are 0 when $a \notin P(V) \cap \mathbb{I}$ and are $(L(-1) u)($ wt $u+a) x^{- \text {wt } u-a-1},(-$ wt $u-a) u($ wt $u+a-1) x^{- \text {wt } u-a-1}$ and $(\mathcal{N} u)($ wt $u+a-1) x^{- \text {wt } u-a-1}$, respectively, when $a \in P(V) \cap \mathbb{I}$. Then in $S_{g}^{1}(M)$, for $a \in P(V) \cap \mathbb{I}$,

$$
\begin{aligned}
& \pi_{M} Y_{0}^{t}(L(-1) u, x) w-\pi_{M} \frac{d}{d x} Y_{0}^{t}(u, x) w+\pi_{M} x^{-1} Y_{0}^{t}(\mathcal{N} u, x) w \\
& \quad=((L(-1) u)(\text { wt } u+a)+(\text { wt } u+a) u(\text { wt } u+a-1)+(\mathcal{N} u)(\text { wt } u+a-1)) w x^{-\mathrm{wt} u-a-1} \\
& \quad=o_{t}((L(-1)+L(0)+a+\mathcal{N}) u) w x^{-\mathrm{wt} u-a-1} \\
& \quad=o_{t}\left((L(-1)+L(0)+a+\mathcal{N}) \mathcal{U}(1)(\mathcal{U}(1))^{-1} u\right) w x^{-\mathrm{wt} u-a-1} .
\end{aligned}
$$

By (1.15) in [H2] $\left((L(-1)+L(0)) \mathcal{U}(1)=\mathcal{U}(1) \frac{L(-1)}{2 \pi \sqrt{-1}}\right)$ and the fact that the Virasoro operators commutes with $\mathcal{N}$, we have

$$
(L(-1)+L(0)+a+\mathcal{N}) \mathcal{U}(1)=\mathcal{U}(1)\left(\frac{L(-1)}{2 \pi \sqrt{-1}}+a+\mathcal{N}\right) .
$$

Thus the right-hand side of (5.15) is equal to

$$
\begin{aligned}
& o_{t}\left(\mathcal{U}(1)\left(\frac{L(-1)}{2 \pi \sqrt{-1}}+a+\mathcal{N}\right)(\mathcal{U}(1))^{-1} u\right) w x^{-\mathrm{wt} u-a-1} \\
& \quad=\rho\left(\left(\frac{L(-1)}{2 \pi \sqrt{-1}}+a+\mathcal{N}\right)(\mathcal{U}(1))^{-1} u+\tilde{O}_{g}(V)\right) w x^{-\mathrm{wt} u-a-1}
\end{aligned}
$$

Since $\left(\frac{L(-1)}{2 \pi \sqrt{-1}}+a+\mathcal{N}\right) v \in \tilde{O}_{g}(V)$ for $v \in V$ (by Theorem 4.1) and $\rho\left(u+\tilde{O}_{g}(V)\right)=0$ for $u \in \tilde{O}_{g}(V)$, the right-hand side of (5.14) and thus the right-hand side of (5.15) are 0 in $S_{g}^{1}(M)$, proving (5.11) in the case $w \in M$.

For the same $u$ and $v$ but for $w \in S_{g}^{1}(V)_{+}$, by straightforward calculations using the commutator formula for $Y_{0}^{t}$, the properties of the formal $\delta$-function and Res and the $L(-1)$ derivative property for $Y$, it is easy to show that

$$
Y_{0}^{t}(L(-1) u, x) w-\frac{d}{d x} Y_{0}^{t}(u, x) w+x^{-1} Y_{0}^{t}(\mathcal{N} u, x)
$$

commutes with $Y_{0}^{t}(v, y)$ for $v \in V$ and thus also commutes with $v(n)$ for $v \in V^{[b+\mathbb{Z}]}, b \in P(V)$ and $n \in b+\mathbb{Z}$. Here we omit the calculations. Now let $w=v_{1}\left(m_{1}\right) \cdots v_{k}\left(m_{k}\right) w_{0} \in S_{g}^{1}(M)_{+}$ 
for $v_{i} \in V^{\left[a_{i}+\mathbb{Z}\right]}, m_{i} \in a_{i}+\mathbb{Z}, w_{0} \in M$ such that wt $v_{i}-m_{i}-1>0$. Then

$$
\begin{aligned}
& \pi_{M}\left(Y_{0}^{t}(L(-1) u, x) w-\frac{d}{d x} Y_{0}^{t}(u, x) w+x^{-1} Y_{0}^{t}(\mathcal{N} u, x)\right) w \\
& \quad=\pi_{M}\left(Y_{0}^{t}(L(-1) u, x) w-\frac{d}{d x} Y_{0}^{t}(u, x) w+x^{-1} Y_{0}^{t}(\mathcal{N} u, x)\right) v_{1}\left(m_{1}\right) \cdots v_{k}\left(m_{k}\right) w_{0} \\
& \quad=\pi_{M} v_{1}\left(m_{1}\right) \cdots v_{k}\left(m_{k}\right)\left(Y_{0}^{t}(L(-1) u, x) w-\frac{d}{d x} Y_{0}^{t}(u, x) w+x^{-1} Y_{0}^{t}(\mathcal{N} u, x)\right) w_{0} .
\end{aligned}
$$

Since the weight of $v_{1}\left(m_{1}\right) \cdots v_{k}\left(m_{k}\right)$ is bigger than 0 and $w_{0} \in M$, the right-hand side of (5.15) must be 0, proving (5.11) in this case.

We now prove (5.12) in the case $w \in M$. To prove (5.12) in this case, we need only prove that coefficients in $x_{0}$ of the two sides of (5.12) are equal, that is, for $n \in \mathbb{Z}$,

$$
\begin{aligned}
& \operatorname{Res}_{x_{0}} x_{0}^{n}\left(x_{0}+x_{2}\right)^{l(u)} \pi_{M} Y_{0}^{t}\left(u, x_{0}+x_{2}\right) Y_{0}^{t}\left(v, x_{2}\right) w \\
& \quad=\operatorname{Res}_{x_{0}} x_{0}^{n}\left(x_{2}+x_{0}\right)^{l(u)} \pi_{M} Y_{0}^{t}\left(Y\left(\left(1+\frac{x_{0}}{x_{2}}\right)^{\mathcal{N}} u, x_{0}\right) v, x_{2}\right) w .
\end{aligned}
$$

For $n \in \mathbb{Z}$, by changing the variable from $x_{0}$ to $x_{1}$, we obtain

$$
\begin{aligned}
& \operatorname{Res}_{x_{0}} x_{0}^{n}\left(x_{0}+x_{2}\right)^{l(u)} \pi_{M} Y_{0}^{t}\left(u, x_{0}+x_{2}\right) Y_{0}^{t}\left(v, x_{2}\right) w \\
& \quad=\operatorname{Res}_{x_{1}}\left(x_{1}-x_{2}\right)^{n} x_{1}^{l(u)} \pi_{M} Y_{0}^{t}\left(u, x_{1}\right) Y_{0}^{t}\left(v, x_{2}\right) w .
\end{aligned}
$$

For $n \in \mathbb{N}$, using the commutator formula for $Y_{0}^{t}$ for $S_{g}^{1}(M)$, we see that the right-hand side of (5.17) is equal to

$$
\begin{aligned}
& \operatorname{Res}_{x_{1}}\left(x_{1}-x_{2}\right)^{n} x_{1}^{l(u)} \pi_{M} Y_{0}^{t}\left(v, x_{2}\right) Y_{0}^{t}\left(u, x_{1}\right) w \\
& +\operatorname{Res}_{x_{1}} \operatorname{Res}_{x_{0}}\left(x_{1}-x_{2}\right)^{n} x_{1}^{l(u)} x_{1}^{-1} \delta\left(\frac{x_{2}+x_{0}}{x_{1}}\right)\left(\frac{x_{2}+x_{0}}{x_{1}}\right)^{a} . \\
& \cdot \pi_{M} Y_{0}^{t}\left(Y\left(\left(1+\frac{x_{0}}{x_{2}}\right)^{\mathcal{N}} u, x_{0}\right) v, x_{2}\right) w .
\end{aligned}
$$

Since $u(l(u)) w=0$

$$
\operatorname{Res}_{x_{1}} x_{1}^{l(u)+i} Y_{0}^{t}\left(u, x_{1}\right) w=0
$$

for $0 \leq i \leq n$. Thus the first term in the right-hand side of (5.18) is 0 . The second term in the right-hand side of (5.18) is equal to

$$
\operatorname{Res}_{x_{0}} x_{0}^{n}\left(x_{2}+x_{0}\right)^{l(u)} \pi_{M} Y_{0}^{t}\left(Y\left(\left(1+\frac{x_{0}}{x_{2}}\right)^{\mathcal{N}} u, x_{0}\right) v, x_{2}\right) w
$$

From (5.18) and (5.19), we obtain (5.16) in the case $n \in \mathbb{N}$. 
For $n \in-\mathbb{Z}_{+}-1, \operatorname{Res}_{x_{1}}\left(x_{1}-x_{2}\right)^{n} x_{1}^{l(u)} Y_{0}^{t}\left(u, x_{1}\right)$ contains terms proportional to $u(l(u)+n-i)$ for $i \in \mathbb{N}$. Recall from Section 4 that $l(u)=$ wt $u+a$ when $a \in \mathbb{I}$ and $l(u)=a+$ wt $u-1$ when $a \in P(V) \cap((0,1)+\mathbb{I})$. Together with $n \in-\mathbb{Z}_{+}-1$ and $i \in \mathbb{N}$, we have $\pi_{M} u(l(u)+$ $n-i) v(k) w=0$ for $k \in \mathbb{C}$. Thus the right-hand side of (5.17) is 0 . We now show that the right-hand side of (5.16) is also 0 . First note that for homogeneous $u \in V^{[a+\mathbb{Z}]}$ and $w \in M$, $\pi_{M} Y_{0}^{g}\left(x^{L(0)} u, x\right) w=u($ wt $u+a-1) w x^{-a}=o_{t}(u) w x^{-a}$ if $a \in P(V) \cap \mathbb{I}$ and is 0 if otherwise. Now come back to $u, v, w$ as above. Recall the operator $\mathbf{L}$ introduced in Section 3. By changing the variable $x_{0}$ to $y=\frac{x_{0}}{x_{2}}$, we obtain

$$
\begin{aligned}
\operatorname{Res}_{x_{0}} x_{0}^{n}\left(x_{2}+x_{0}\right)^{l(u)} \pi_{M} Y_{0}^{t}\left(Y\left(\left(1+\frac{x_{0}}{x_{2}}\right)^{\mathcal{N}} u, x_{0}\right) v, x_{2}\right) w \\
=\operatorname{Res}_{y} x_{2}\left(x_{2} y\right)^{n}\left(x_{2}+x_{2} y\right)^{l(u)} \pi_{M} Y_{0}^{t}\left(Y\left((1+y)^{\mathcal{N}} u, x_{2} y\right) v, x_{2}\right) w \\
=x_{2}^{l(u)+n+1+\mathrm{wt} u+\operatorname{wt} v} \pi_{M} Y_{0}^{t}\left(x_{2}^{L(0)} \operatorname{Res}_{y} y^{n} Y\left((1+y)^{\mathbf{L}+\mathcal{N}} u, y\right) v, x_{2}\right) w \\
=x_{2}^{l(u)+n+1+\operatorname{wt} u+\operatorname{wt} v}{ }_{o}\left(\operatorname{Res}_{y} y^{n} Y\left((1+y)^{\mathbf{L}+\mathcal{N}} u, y\right) v\right) w \\
=x_{2}^{l(u)+n+1+\operatorname{wt} u+\operatorname{wt} v} \rho\left(\mathcal{U}(1)^{-1} \operatorname{Res}_{y} y^{n} Y\left((1+y)^{\mathbf{L}+\mathcal{N}} u, y\right) v+\tilde{O}_{g}(V)\right) w .
\end{aligned}
$$

By (4.7) and (4.1),

$$
\begin{aligned}
\mathcal{U}(1)^{-1} \operatorname{Res}_{y} y^{n} Y\left((1+y)^{\mathbf{L}+\mathcal{N}} u, y\right) v \\
\quad=\operatorname{Res}_{y} y^{n} Y\left((1+y)^{\mathbf{L}-L(0)+\mathcal{N}} \mathcal{U}(1)^{-1} u, \frac{1}{2 \pi \sqrt{-1}} \log (1+y)\right) \mathcal{U}(1)^{-1} v \\
\quad \in \tilde{O}_{g}(V) .
\end{aligned}
$$

Thus the right-hand side of (5.20) is 0 .

We still need to prove (5.16) in the case $n=-1$. We first prove this formula when $a \in P(V) \cap((0,1)+\mathbb{I})$. Since $l(u)=$ wt $u+a-1$ and $\pi_{M} u(l(u)-1-i) v(k) w=\pi_{M} u($ wt $u+$ $a-2-i) v(k) w=0$ for $i \in \mathbb{N}$ and $k \in \mathbb{C}$,

$$
\begin{aligned}
& \operatorname{Res}_{x_{0}} x_{0}^{-1}\left(x_{0}+x_{2}\right)^{l(u)} \pi_{M} Y_{0}^{t}\left(u, x_{0}+x_{2}\right) Y_{0}^{t}\left(v, x_{2}\right) w \\
& \quad=\operatorname{Res}_{x_{1}}\left(x_{1}-x_{2}\right)^{-1} x_{1}^{l(u)} \pi_{M} Y_{0}^{t}\left(u, x_{1}\right) Y_{0}^{t}\left(v, x_{2}\right) w \\
& \quad=0
\end{aligned}
$$

On the other hand, the same calculation as in (5.20) and (5.21) gives

$$
\begin{aligned}
& \operatorname{Res}_{x_{0}} x_{0}^{-1}\left(x_{2}+x_{0}\right)^{l(u)} \pi_{M} Y_{0}^{t}\left(Y\left(\left(1+\frac{x_{0}}{x_{2}}\right)^{\mathcal{N}} u, x_{0}\right) v, x_{2}\right) w \\
& \quad=x_{2}^{l(u)+\operatorname{wt} u+\operatorname{wt} v} \rho\left(\mathcal{U}(1)^{-1} \operatorname{Res}_{y} y^{-1} Y\left((1+y)^{\mathbf{L}+\mathcal{N}} u, y\right) v+\tilde{O}_{g}(V)\right) w
\end{aligned}
$$




$$
\begin{aligned}
& =x_{2}^{l(u)+\mathrm{wt} u+\mathrm{wt} v} \rho\left(\operatorname { R e s } _ { y } y ^ { - 2 } Y \left(y^{L(0)-\mathbf{L}+\mathbf{A}}(1+y)^{\mathbf{L}-L(0)+\mathcal{N}}\right.\right. \\
& \left.\left.\qquad \mathcal{U}(1)^{-1} u, \frac{1}{2 \pi \sqrt{-1}} \log (1+y)\right) \mathcal{U}(1)^{-1} v+\tilde{O}_{g}(V)\right) w \\
& =0
\end{aligned}
$$

because $a \in P(V) \cap((0,1)+\mathbb{I})$, proving (5.16) $)$ in this case $(n=-1$ and $a \in P(V) \cap((0,1)+\mathbb{I}))$.

In the case $a \in P(V) \cap \mathbb{I}$, since $l(u)=$ wt $u+a$, we have $\pi_{M} u(l(u)-1-i) v(k) w=$ $\pi_{M} u($ wt $u+a-1-i) v(k) w=0$ for $i \in \mathbb{Z}_{+}$and $k \in \mathbb{C}, \pi_{M} u(l(u)-1) v(k) w=0$ for either $v \in V^{[b]}, b \in P(V) \cap((0,1)+\mathbb{I}), k \in \mathbb{C}$ or $v \in V^{[b]}, b \in P(V) \cap \mathbb{I}, k \neq$ wt $v+b-1$ and $\pi_{M} u(l(u)-1) v($ wt $v+b-1) w=u($ wt $u+a-1) v($ wt $v+b-1) w$ for $v \in V^{[b]}, b \in P(V) \cap \mathbb{I}$. Using these formulas, we see that in the case $v \in V^{[b]}$ and $b \in P(V) \cap((0,1)+\mathbb{I})$, the right-hand side of (5.17) is equal to 0 . But in this case,

$$
Y_{0}^{t}\left(Y\left(\left(1+\frac{x_{0}}{x_{2}}\right)^{\mathcal{N}} u, x_{0}\right) v, x_{2}\right) w=0 .
$$

Thus (5.16) holds in this case $(n=-1, a \in P(V) \cap \mathbb{I}$ and $b \in P(V) \cap((0,1)+\mathbb{I}))$. In the case $v \in V^{[b]}$ and $b \in P(V) \cap \mathbb{I}$, the right-hand side of (5.17) is equal to

$$
\begin{array}{rl}
x_{2}^{-\mathrm{wt}} & v(\mathrm{wt} u+a-1) v(\mathrm{wt} v+b-1) w \\
= & x_{2}^{-\mathrm{wt} v} o_{t}(u) o_{t}(v) w \\
= & x_{2}^{-\mathrm{wt} v} \rho\left(\mathcal{U}(1)^{-1} u+\tilde{O}_{g}(V)\right) \rho\left(\rho\left(\mathcal{U}(1)^{-1} v+\tilde{O}_{g}(V)\right) w\right. \\
= & x_{2}^{-\mathrm{wt} v} \rho\left(\left(\mathcal{U}(1)^{-1} u \bullet g\left(\mathcal{U}(1)^{-1} v\right)+\tilde{O}_{g}(V)\right) w\right. \\
= & x_{2}^{-\mathrm{wt} v} \rho\left(\mathcal{U}(1)^{-1}\left(u *_{g} v\right)+\tilde{O}_{g}(V)\right) w \\
= & x_{2}^{-\mathrm{wt} v} o_{t}\left(u *_{g} v\right) w \\
= & x_{2}^{-\mathrm{wt} v} \pi_{M} Y_{0}^{t}\left(x_{2}^{L(0)}\left(u *_{g} v\right), x_{2}\right) w \\
=x_{2}^{-\mathrm{wt}}{ }^{v} \operatorname{Res}_{y} y^{-1} \pi_{M} Y_{0}^{t}\left(x_{2}^{L(0)} Y\left((1+y)^{L(0)+\mathcal{N}} u, y\right) v, x_{2}\right) w \\
=\operatorname{Res}_{x_{0}} x_{0}^{-1} x_{2}^{-\mathrm{wt} v} \pi_{M} Y_{0}^{t}\left(x_{2}^{L(0)} Y\left(\left(1+\frac{x_{0}}{x_{2}}\right)^{L(0)+\mathcal{N}} u, \frac{x_{0}}{x_{2}}\right) v, x_{2}\right) w \\
=\operatorname{Res}_{x_{0}} x_{0}^{-1}\left(x_{2}+x_{0}\right)^{l} \pi_{M} Y_{0}^{t}\left(Y\left(\left(1+\frac{x_{0}}{x_{2}}\right)^{\mathcal{N}} u, x_{0}\right) v, x_{2}\right) w,
\end{array}
$$

proving (5.16) in the case $n=-1, a, b \in P(V) \cap \mathbb{I}$.

We now prove (5.12) for the same $u$ and $v$ but for $w \in S_{g}^{1}(V)_{+}$. By straightforward calculations generalizing the last part of the calculations in the proof of Proposition 6.1 in [DLM1, for $u \in V^{[a+\mathbb{Z}]}, a \in P(V), v \in V, v_{1} \in V^{\left[a_{1}+\mathbb{Z}\right]}, a_{1} \in P(V), m_{1} \in a_{1}+\mathbb{Z}$ such that 
$\Re\left(\right.$ wt $\left.v_{1}-m_{1}-1\right)>0, w \in S_{g}^{1}(V), l \in a+\mathbb{Z}$ such that $u(n) v_{1}\left(m_{1}\right) w=0$ for $n \in a+\mathbb{Z}$ and $n-a \geq l-a$, we have

$$
\begin{aligned}
& \left(\left(x_{0}+x_{2}\right)^{l} Y_{0}^{t}\left(u, x_{0}+x_{2}\right) Y_{0}^{t}\left(v, x_{2}\right)\right. \\
& \left.-\left(x_{2}+x_{0}\right)^{l} Y_{0}^{t}\left(Y\left(\left(1+\frac{x_{0}}{x_{2}}\right)^{\mathcal{N}} u, x_{0}\right) v, x_{2}\right)\right) v_{1}\left(m_{1}\right) w \\
& =v_{1}\left(m_{1}\right)\left(\left(x_{0}+x_{2}\right)^{l} Y_{0}^{t}\left(u, x_{0}+x_{2}\right) Y_{0}^{t}\left(v, x_{2}\right)\right. \\
& \left.\quad-\left(x_{2}+x_{0}\right)^{l} Y_{0}^{t}\left(Y\left(\left(1+\frac{x_{0}}{x_{2}}\right)^{\mathcal{N}} u, x_{0}\right) v, x_{2}\right)\right) w
\end{aligned}
$$

Here we omit the detailed calculations. Now let $w=v_{1}\left(m_{1}\right) \cdots v_{k}\left(m_{k}\right) w_{0}$ where $v_{i} \in V^{\left[a_{i}+\mathbb{Z}\right]}$, $m_{i} \in a_{i}+\mathbb{Z}$ satisfying $\Re\left(\right.$ wt $\left.v_{i}-m_{i}-1\right)>0$ and $w_{0} \in M$. Then by (5.24), for $l \in a+\mathbb{Z}$ such that $u(n) v_{1}\left(m_{1}\right) \cdots v_{k}\left(m_{k}\right) w_{0}=0$ for $n \in a+\mathbb{Z}$ and $n-a \geq l-a$, we have

$$
\begin{aligned}
& \pi_{M}\left(\left(x_{0}+x_{2}\right)^{l} Y_{0}^{t}\left(u, x_{0}+x_{2}\right) Y_{0}^{t}\left(v, x_{2}\right)\right. \\
& \left.-\left(x_{2}+x_{0}\right)^{l} Y_{0}^{t}\left(Y\left(\left(1+\frac{x_{0}}{x_{2}}\right)^{\mathcal{N}} u, x_{0}\right) v, x_{2}\right)\right) v_{1}\left(m_{1}\right) \cdots v_{k}\left(m_{k}\right) w_{0} \\
& =\pi_{M} v_{1}\left(m_{1}\right) \cdots v_{k}\left(m_{k}\right)\left(\left(x_{0}+x_{2}\right)^{l} Y_{0}^{t}\left(u, x_{0}+x_{2}\right) Y_{0}^{t}\left(v, x_{2}\right)\right. \\
& \left.\quad-\left(x_{2}+x_{0}\right)^{l} Y_{0}^{t}\left(Y\left(\left(1+\frac{x_{0}}{x_{2}}\right)^{\mathcal{N}} u, x_{0}\right) v, x_{2}\right)\right) w_{0} \\
& =0
\end{aligned}
$$

where the last step uses the fact that $\Re\left(\right.$ wt $\left.v_{i}-m_{i}-1\right)>0$ for $i=1, \ldots, k$ together with the projection $\pi_{M}$ force us to take only components of

$$
\left(x_{0}+x_{2}\right)^{l} Y_{0}^{t}\left(u, x_{0}+x_{2}\right) Y_{0}^{t}\left(v, x_{2}\right)-\left(x_{2}+x_{0}\right)^{l} Y_{0}^{t}\left(Y\left(\left(1+\frac{x_{0}}{x_{2}}\right)^{\mathcal{N}} u, x_{0}\right) v, x_{2}\right)
$$

of negative weights. This proves (5.12) in the case that $w \in S_{g}^{1}(V)_{+}$.

Let $W$ be a $\overline{\mathbb{C}}_{+}$-graded weak $g$-twisted $V$-module. We define a linear map from $S_{g}\left(\Omega_{g}(W)\right)$ to $W$ by mapping $u_{1}\left(m_{1}\right) \cdots u_{k}\left(m_{k}\right) w$ of $S_{g}(M)$ to $u_{1}\left(m_{1}\right) \cdots u_{k}\left(m_{k}\right) w$ of $W$ for $u_{i} \in V^{\left[a_{i}+\mathbb{Z}\right]}$, $m_{i} \in a_{i}+\mathbb{Z}(i=1, \ldots, k)$ and $w \in \Omega_{g}(W)$. Note that the only relations among $u_{1}\left(m_{1}\right) \cdots u_{k}\left(m_{k}\right) w$ for $u_{i} \in V, m_{i} \in \mathbb{Z}(i=1, \ldots, k)$ and $w \in \Omega_{g}(W)$ are given by the action of $V$ on $\Omega_{g}(W)$, the commutator formula, the weak associativity and the $L(-1)$-derivative property for $Y_{0}^{g}$. 
These relations also hold in $W$. Thus this map is well-defined. Clearly, this is a surjective homomorphism of $\overline{\mathbb{C}}_{+}$-graded weak $g$-twisted $V$-modules from $S_{g}\left(\Omega_{g}(W)\right)$ to $\overline{\mathbb{C}}_{+}$-graded weak $g$-twisted $V$-submodule of $W$ generated by $\Omega_{g}(W)$.

The other statements follow immediately.

\section{Isomorphisms between $Z_{g}(V), \tilde{A}_{g}(V)$ and $A_{g}(V)$}

In this section, using the results obtained in the preceding sections, we prove that the algebras $Z_{g}(V), \tilde{A}_{g}(V)$ and $A_{g}(V)$ are isomorphic to each other.

Theorem 6.1 The associative algebras $Z_{g}(V)$ and $A_{g}(V)$ are isomorphic.

Proof. We view $A_{g}(V)$ as an $A_{g}(V)$-module. By Theorem [5.6, $S_{g}\left(A_{g}(V)\right)$ is a $\overline{\mathbb{C}}_{+}$-graded $g$-twisted $V$-module such that $\Omega_{g}\left(S_{g}\left(A_{g}(V)\right)\right)=A_{g}(V)$. Then by Proposition 5.2, $A_{g}(V)$ is a $Z_{g}(V)$-module and, by definition, the action of $Z_{g}(V)$ on $A_{g}(V)$ is given by the homomorphism from $Z_{g}(V)$ to the endomorphism algebra of $A_{g}(V)$ determined by $\left[o_{g}(v)\right] \mapsto v+O_{g}(V)$ for $v \in V$, where $v+O_{g}(V)$ is viewed as an element of the endomorphism algebra of $A_{g}(V)$. Here we have also used Proposition 3.7. Since the image of this homomorphsim is in fact in $A_{g}(V)$, it is a homomorphism $f: Z_{g}(V) \rightarrow A_{g}(V)$ of associative algebras.

Conversely, we view $Z_{g}(V)$ as a $Z_{g}(V)$-module. By Theorem [5.3, $H_{g}\left(Z_{g}(V)\right)$ is a $\overline{\mathbb{C}}_{+^{-}}$ graded $g$-twisted $V$-module such that $\Omega_{g}\left(H_{g}\left(Z_{g}(V)\right)\right)=Z_{g}(V)$. Then by Theorem 5.5, $Z_{g}(V)$ is an $A_{g}(V)$-module and, by definition, the action of $A_{g}(V)$ on $Z_{g}(V)$ is given by the homomorphism from $A_{g}(V)$ to the endomorphism algebra of $Z_{g}(V)$ defined by $v+O_{g}(V) \mapsto$ $\left[o_{g}(v)\right]$ for $v \in V$, where $\left[o_{g}(v)\right]$ is viewed as an element of the endomorphism algebra of $Z_{g}(V)$. Since the image of this homomorphsim is in fact in $Z_{g}(V)$, it is a homomorphism $g: A_{g}(V) \rightarrow Z_{g}(V)$ of associative algebras.

Clearly $f$ and $g$ are inverse to each other. Thus they are isomorphisms of associative algebras.

Corollary 6.2 The associative algebras $Z_{g}(V)$ and $\tilde{A}_{g}(V)$ are isomorphic.

From Theorem 6.1, we also obtain an improvement of Proposition 3.7;

Corollary 6.3 The elements of $Z_{g}(V)$ are of the form $\left[o_{g}(v)\right]$ for $v \in V$.

Proof. Since an element of $A_{g}(V)$ is of the form $v+O_{g}(V)$ for $v \in V$ and under the isomorphism from $A_{g}(V)$ to $Z_{g}(V)$, such an element is mapped to $\left[o_{g}(v)\right]$ for $v \in V$, the conclusion follows. 
Remark 6.4 Because of Theorem 6.1, if we have results for the algebra $Z_{g}(V)$ (or $A_{g}(V)$ ), we can immediately conclude that the same results hold for $A_{g}(V)$ (or $Z_{g}(V)$ ). In particular, we can derive the results in Section 5 on $A_{g}(V)$ (or $Z_{g}(V)$ ) from the corresponding results on $Z_{g}(V)$ (or $A_{g}(V)$ ). But since our proof of Theorem 6.1 has used the results in Section 5 for both $Z_{g}(V)$ and $A_{g}(V)$, the proofs of the results in Section 5 are all needed.

Remark 6.5 In many cases, using Corollary [6.3, it is easier to calculate $Z_{g}(V)$ than to calculate $A_{g}(V)$ or $\tilde{A}_{g}(V)$. The algebra $\tilde{A}_{g}(V)$ is more natural for the study of modular invariance. The algebra $A_{g}(V)$ is in some sense a bridge between $Z_{g}(V)$ and $\tilde{A}_{g}(V)$. We expect that $Z_{g}(V)$ will play an important role in the future construction and study of twisted modules and twisted intertwining operators.

\section{References}

[B] B. Bakalov, Twisted logarithmic modules of vertex algebras, to appear; arXiv:1504.06381

[BN] D. Brungs and W. Nahm, The associative algebras of conformal field theory, Lett. Math. Phys. 47 (1999), 379-383.

[DLM1] C. Dong, H. Li and G. Mason, Twisted representations of vertex operator algebras, Math. Ann. 310 (1998), 571-600.

[DLM2] C. Dong, H. Li and G. Mason, Vertex operator algebras and associative algebras, J. Algebra 206 (1998), 67-96.

[FLM] I. B. Frenkel, J. Lepowsky and A. Meurman, Vertex Operator Algebras and the Monster, Pure and Appl. Math., Vol. 134, Academic Press, Boston, 1988.

[FZ] I. B. Frenkel and Y. Zhu, Vertex operator algebras associated to representations of affine and Virasoro algebras, Duke Math. J. 66 (1992), 123-168.

[H1] Y.-Z. Huang, Generalized rationality and a Jacobi identity for intertwining operator algebras, Selecta Math. 6 (2000), 225-267.

[H2] Y.-Z. Huang, Differential equations, duality and modular invariance, Comm. Contemp. Math. 7 (2005), 649-706.

[H3] Y.-Z. Huang, Generalized twisted modules associated to general automorphisms of a vertex operator algebra, Comm. Math. Phys. 298 (2010), 265-292.

[HLZ] Y.-Z. Huang, J. Lepowsky and L. Zhang, Logarithmic tensor category theory, V: Convergence condition for intertwining maps and the corresponding compatibility condition, to appear; arXiv:1012.4199. 
[HY] Y.-Z. Huang and J. Yang, On functors between module categories for associative algebras and for $\mathbb{N}$-graded vertex algebras, J. Alg. 409 (2014), 344-361.

[NT] K. Nagatomo and A. Tsuchiya, Conformal field theories associated to regular chiral vertex operator algebras, I: Theories over the projective line, Duke Math. J. 128 (2005), 393-471.

[T] V. Turaev, Homotopy field theory in dimension 3 and crossed group-categories, arxiv:math.GT/0005291.

[Z] Y. Zhu, Modular invariance of characters of vertex operator algebras, J. Amer. Math. Soc. 9 (1996), 237-307.

Department of Mathematics, Rutgers University, 110 Frelinghuysen Rd., PiscatAWAY, NJ 08854-8019

E-mail address: yzhuang@math.rutgers.edu

Department of Mathematics, University of Notre Dame, 278 Hurley Building, Notre DAME, IN 46556

E-mail address: jyang7@nd.edu 